\title{
Prefrontal Dynamics Associated with Efficient Detours and Shortcuts: A Combined Functional Magnetic Resonance Imaging and Magnetoencenphalography Study
}

\author{
Amir-Homayoun Javadi ${ }^{1,2 *}$, Eva Zita Patai ${ }^{1 *}$, Eugenia Marin-Garcia ${ }^{3}$, Aaron Margolis ${ }^{1}$, \\ Heng-Ru M. Tan ${ }^{1}$, Dharshan Kumaran ${ }^{1,4}$, Marko Nardini ${ }^{5}$, Will Penny ${ }^{6}$, \\ Emrah Duzel $^{7}$, Peter Dayan ${ }^{8}$, and Hugo J. Spiers ${ }^{1}$
}

\begin{abstract}
Central to the concept of the "cognitive map" is that it confers behavioral flexibility, allowing animals to take efficient detours, exploit shortcuts, and avoid alluring, but unhelpful, paths. The neural underpinnings of such naturalistic and flexible behavior remain unclear. In two neuroimaging experiments, we tested human participants on their ability to navigate to a set of goal locations in a virtual desert island riven by lava, which occasionally spread to block selected paths (necessitating detours) or receded to open new paths (affording real shortcuts or false shortcuts to be avoided).
\end{abstract}

Detours activated a network of frontal regions compared with shortcuts. Activity in the right dorsolateral PFC specifically increased when participants encountered tempting false shortcuts that led along suboptimal paths that needed to be differentiated from real shortcuts. We also report modulation in event-related fields and theta power in these situations, providing insight to the temporal evolution of response to encountering detours and shortcuts. These results help inform current models as to how the brain supports navigation and planning in dynamic environments.

\section{INTRODUCTION}

A challenge all motile animals face is adapting to changes in an environment so that they can efficiently return to safety or find food. Adaptations include identifying novel shortcuts and minimizing the lengths of imposed detours. Tolman (1948) conceptualized this ability as arising from an internal "cognitive map" (or, in control theoretical terms, an internal model) of the environment. Evidence from electrophysiological recordings in rodents and functional magnetic resonance imaging (fMRI) in humans has supported the view that hippocampus contains a cognitive map (Ekstrom, Spiers, Bohbot, \& Rosenbaum, 2018; Epstein, Patai, Julian, \& Spiers, 2017; Spiers \& Barry, 2015; O'Keefe \& Nadel, 1978). However, our knowledge is incomplete of the neural dynamics associated with the use of cognitive maps to solve navigation problems in environments where the path structure of the environment is subject to change.

\footnotetext{
${ }^{1}$ University College London, ${ }^{2}$ University of Kent, ${ }^{3}$ University of the Basque Country (UPV/EHU), ${ }^{4}$ Google Deepmind, London, ${ }^{5}$ Durham University, ${ }^{6}$ University of East Anglia, ${ }^{7}$ University Hospital Magdeburg, ${ }^{8}$ Max Planck Institute for Biological Cybernetics

*These authors contributed equally to this work.
}

Early studies with rats (Tolman \& Honzik, 1930), along with more recent studies in both rats and other mammals (Alvernhe, Save, \& Poucet, 2011; Winocur, Moscovitch, Rosenbaum, \& Sekeres, 2010; Alvernhe, Van Cauter, Save, \& Poucet, 2008; Chapuis, 1987; Chapuis, Durup, \& Thinus-Blanc, 1987; Poucet, Thinus-Blanc, \& Chapuis, 1983), have helped characterize flexible navigation behavior when the environmental layout changes. Furthermore, electrophysiological recording of hippocampal place cells has revealed "remapping" in response to the changes in barriers that induced detours or shortcuts (Alvernhe et al., 2008, 2011; Poucet et al., 1983). However, these studies generally examined neural coding of the new maze geometry (e.g., place cell remapping) rather than the event-related responses evoked by the changes to the maze. Similarly, the possibilities of exploiting cross-species comparisons of the underlying neural mechanisms during navigation in dynamic environments are limited by the observation that, despite extensive research on the neural oscillations that arise during navigation in rodents, few studies of human navigation have examined neural oscillations or evoked responses at a fine-grained timescale in relation to the spatial processing involved (Vass et al., 2016; Kaplan et al., 2014; Cornwell, Johnson, Holroyd, Carver, \& Grillon, 2008).

By contrast, a number of functional neuroimaging studies in humans have studied the evoked responses 
to detours (Howard et al., 2014; Simon \& Daw, 2011; Viard, Doeller, Hartley, Bird, \& Burgess, 2011; Xu, Evensmoen, Lehn, Pintzka, \& Håberg, 2010; Iaria, Fox, Chen, Petrides, \& Barton, 2008; Rauchs et al., 2008; Spiers \& Maguire, 2006; Rosenbaum, Ziegler, Winocur, Grady, \& Moscovitch, 2004; Maguire et al., 1998). Rather than revealing hippocampal activity, these studies have consistently reported increased prefrontal activity. These studies report (i) increased activity in the right lateral prefrontal regions when detecting changes in the environment, (ii) activity in frontopolar cortex when replanning and setting subgoals, and (iii) superior prefrontal cortical activity when processing conflict between route options (Spiers \& Gilbert, 2015). Such responses are consistent with the view that the PFC supports flexible behavior in response to changing affordances in the environment (Spiers, 2008; Shallice, 1982).

However, only a more limited number of these neuroimaging studies included shortcuts as well as detours (Ribas-Fernandes et al., 2011; Simon \& Daw, 2011; Yoshida \& Ishii, 2006). Furthermore, the paradigms deployed were not optimized to disentangle the neural responses to these changes; thus, to date we lack evidence as to how neural systems react to shortcuts and how this compares to their reactions to detours. Because both detours and shortcuts change the path to the goal, it is possible that both events elicit similar neural responses. Alternatively, considering the path options after a forced detour might be more taxing on prefrontal systems than simply spotting a potential shortcut and choosing it. However, selecting a shortcut in the real world often requires consideration of its likely benefit, such as "Will it take me in the right direction?" or "Will it lead me down a cul-de-sac?" Such considerations would also likely make greater demands on the neural systems for navigation (Spiers \& Barry, 2015). Here, during scanning with both fMRI and separately using magnetoencenphalography (MEG), we tested participants' navigation performance in a virtual reality (VR)-based environment ("LavaWorld") in which participants navigated a desert island containing hidden treasure with paths constrained by lava, which had the capacity to recede and open new paths (shortcuts) or spread and close others off (detours).

\section{METHODS}

\section{Participants}

\section{Functional Magnetic Resonance Imaging}

Twenty-two individuals (mean age $=21.8 \pm 2.3$ years, range $=19-27$ years; 14 women) participated. To avoid testing participants with poor navigation skills, participants were administered a questionnaire regarding their navigation abilities/strategies (Santa Barbara Sense of Direction Scale $[\mathrm{SBSDS}]$; mean score $=4.9$, range $=3.7-5.7$ ).

\section{Magnetoencenphalography}

Twenty-five individuals (mean age $=22.5 \pm 3.9$ years, range $=18-31 ; 12$ women) participated. Participants were administered a questionnaire regarding their navigation abilities/strategies (Santa Barbara Sense of Direction Scale; mean score $=5.1$, range $=3.2-6.8$ ). All participants scored within $1 S D$ of the mean provided by a study evaluating the SBSDS against spatial abilities (Hegarty, Montello, Richardson, Ishikawa, \& Lovelace, 2006).

There was no overlap in participants between the fMRI and MEG tasks. All participants had normal to correctedto-normal vision, reported no medical implant containing metal, had no history of neurological or psychiatric condition and color blindness, and did not suffer from claustrophobia. All participants gave written consent to participate in the study in accordance with the BirkbeckUCL Centre for Neuroimaging Ethics Committee. Participants were compensated with a minimum of $\$ 70$ plus an additional £10 reward for good performance during the scan. One fMRI participant was excluded from the final sample because there was severe signal loss from the medial-temporal area in their functional scan.

\section{VR Environment: LavaWorld}

A virtual island maze environment was created using Vizard VR software (WorldViz). The maze was a grid network, consisting of "sand" areas that were walkable and "lava" areas, which were unpassable and as such were like walls in a traditional maze. However, the whole maze layout was flat, so there was visibility into the distance over both sand and lava. This allowed participants to stay oriented in the maze throughout the task. Orientation cues were provided by four unique large objects in the distance. Movement was controlled by four buttons: left, right, forward, and backward. Pressing left, right, or backward moved the participant to the grid square to the left, right, or behind, respectively, and rotated the view accordingly. Similarly, pressing forward moved the participant to the next square along. See Figure 1 for a participant viewpoint at one point in the maze. Participants were tested over 2 days; on Day 1, they were trained on the maze, and on Day 2, they were tested in the fMRI/MEG scanner.

\section{Training}

On the first day, participants were trained on the maze $(25 \times 15$ grid $)$ to find goal locations. During this phase, all goal objects (20 in total, distributed across the maze) were visible at all times, and participants navigated from one to the next based on the currently displayed target object (displayed in the top-right corner of the screen). After 1 hr of training, participants were given a test to establish how well they had learned the object locations. On a blank grid, where only the lava was marked, participants 
Figure 1. LavaWorld. Example view of test environment and current goal object (top right corner). A distal cue is visible (arch), and three others were located at the other cardinal directions. The sand represents the path that can be moved along, whereas the red "lava" blocks in the path. During training, objects were visible across the whole maze, and participants used the controls to move forward, left/right, and backward to collect them, with an arrow guiding them toward the object (in the first of three rounds of training). During the test phase, the objects were not visible and the environment could change momentarily, such that the lava shifted around to close an existing path (detours, top row) or reveal new paths (shortcuts or false shortcuts, bottom row). White dotted boxes are to highlight the changes and were not present during the experiment. See Figure 2 for more examples.



had to place all the objects they remembered. They were given feedback from the experimenter, and if needed, prompts as to the missing objects. This memory test was repeated twice more during the training, after 1.5 and $2 \mathrm{hr}$, during which they were encouraged (and occasionally primed if needed) by the experimenter to remember all locations. At completion, for participants to return for the fMRI/MEG phase on the second day, they had to score at $100 \%$ accuracy in placing the objects.

\section{Navigation Test and fMRI/MEG Scan}

On the test day, participants were given a brief refresher of the maze with the objects. While in the MRI scanner, participants performed the test phase of the experiment. A single trial in the test phase is defined as being informed, which is the new goal object, and then finding the way to and arriving at it - the trial did not end until the participant arrived at the goal location. During the test phase, two things were different from training: (1) target objects were not visible, so participants had to navigate between them based on their memories of the locations, and (2) the positions of the lava could change, blocking some paths and creating new ones. During each journey to an object, one change occurred in the lava layout at a specific location (on average 6.9 steps from the start of the route-per condition mean and range reported: long detour: 6.9 [4-12], short detour: 8.4 [5-15], long shortcut: 5 [2-11], short shortcut: 5.7 [4-9], false away: 7.1 [2-16], false toward: 8.8 [7-10]). The paths varied from 10 to 24 steps, as calculated from the start of the trial before a change happened, and from 5 to 27 steps total if the change is included.

At the point of a change, the screen froze for $4 \mathrm{sec}$ to ensure that participants had an opportunity to detect the change and consider their path options. See Figures 1 and 2 for example schematics of the changes. They could either be detours (when a piece of lava was added to block the current path on the grid, thus forcing the participant to take a new, longer, route to their goal), shortcuts (a piece of lava was removed and replaced with traversable sand, allowing the participant to pick a shorter route), false shortcuts (visually identical to shortcuts, but such that traversing them would increase the net distance to the goal because of the layout of the maze), and a control condition (in which the screen froze, but no lava was added/removed). False shortcuts came in two classes: false shortcuts toward and false shortcuts away from the goal, depending on whether or not traversing them would appear to move closer to the goal. For detours and shortcuts, there were also two levels of change to the (optimal) new path, either four or eight grid steps extra/less, respectively. Finally, there were control "follow" trials, which started with an arrow that indicated the direction to travel. In this case, participants were required to follow the twists and turns of the arrow until a new target object appeared. The comparison of "navigation" versus "follow" movements allowed us to relate our results to those of previous experiments (Patai et al., 2019; Javadi et al., 2017; Howard et al., 2014). 


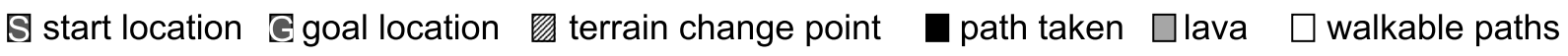
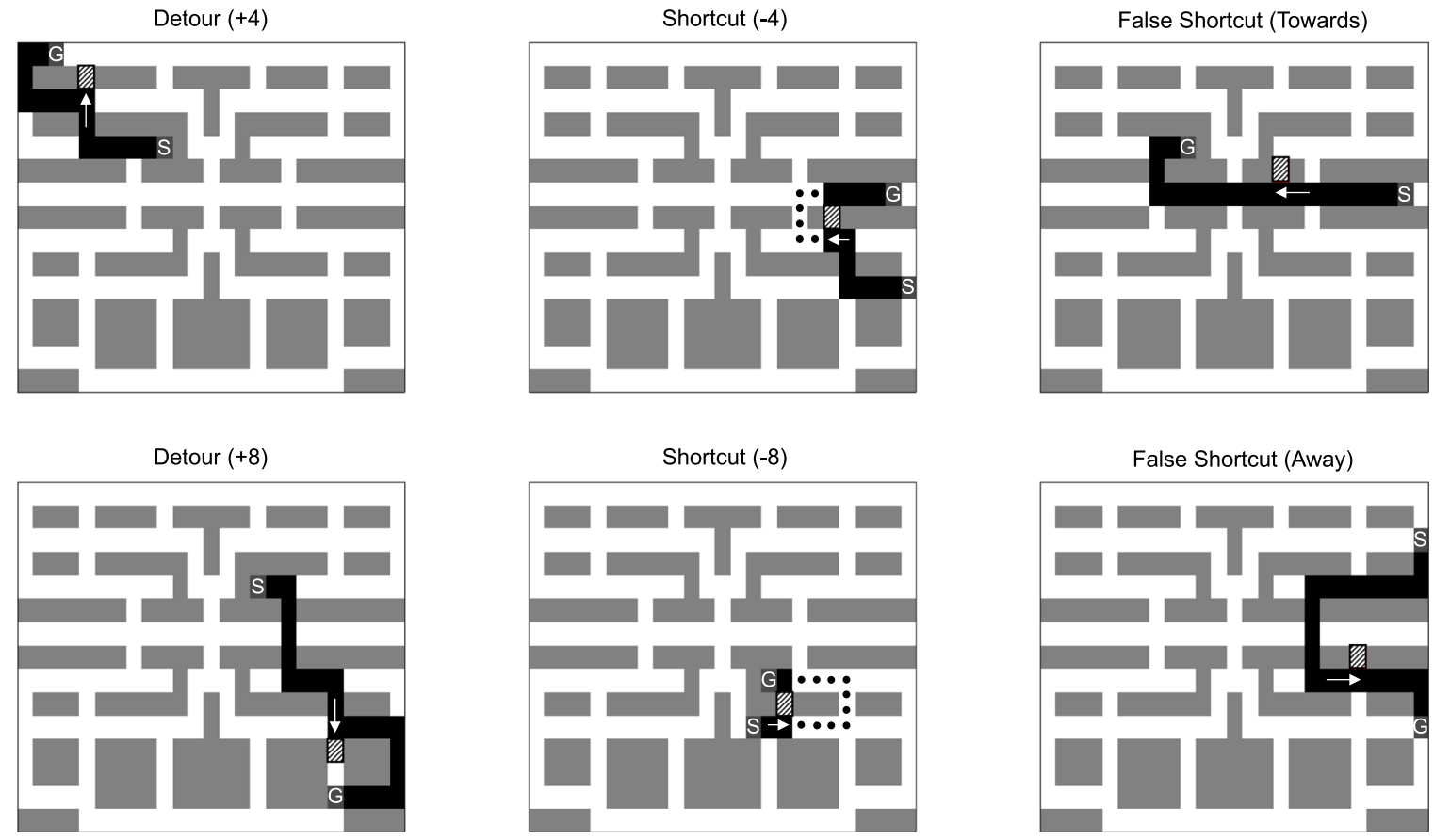

Figure 2. Examples of changes that occurred during routes to the goal. Participants start their path from the last object they found and go toward the current (new) goal location along the shortest path available. For detours, at some point along the route, the participant's path is blocked, and they are forced to take a detour around the lava to reach their goal. In the case of a shortcut, a grid point would be unblocked, thus revealing a novel, shorter route to the goal (originally optimal path shown in dots if no shortcut had been presented). In the case of false shortcuts, taking this opening would be detrimental as it would lead to a longer path to the goal, despite the path seeming to head toward (or away) from it. The full grid was $25 \times 15$ squares and is shown from above in these examples; \pm 4 or 8 refers to the amount added or subtracted in steps.

Before scanning, participants were allowed to familiarize themselves with the scanner button pad and with the changes that would occur. This involved presenting them with a novel environment that had not been experienced on Day 1 and that had no objects, different distal cues and a different maze layout to avoid any confounds or confusion with training and test mazes. Participants could then practice the task in this new environment and accustom themselves to the controls (button pad with four active buttons: left, right, forward, and turn around) and to the appearance of changes to the lava. Note that during training, the future trajectories that could happen after change events (detours, shortcuts, etc.) were not possible to experience. In other words, a stable map with possible routes (sand) and blocks (lava) were experienced only, which then changed during the test phase. The optimal routes between various target locations was thus experienced (assuming no changes in the environment), but these were not presented in the same order during training and test.

\section{fMRI Scanning and Preprocessing}

Scanning was conducted at the Birkbeck-UCL Centre for Neuroimaging using a 1.5-T Siemens Avanto MRI scanner
(Siemens Medical System) with a 32-channel head coil. Each experimental session lasted around $60 \mathrm{~min}$ and was separated in three parts (each of approximately 15-20 min). Approximately 980 functional scans were acquired per session (depending on routes taken), using a gradient-echo incremental EPI sequence (repetition time $[\mathrm{TR}]=3400 \mathrm{msec}$, echo time $[\mathrm{TE}]=50 \mathrm{msec}$, acquisition time $=3315 \mathrm{sec}$, flip angle $=90^{\circ}$, slice tilt $=30^{\circ}$ ). The slice thickness was $2 \mathrm{~mm}$ with a gap of $1 \mathrm{~mm}$. The field of view was $192 \mathrm{~mm}$, and the matrix size was $64 \times 64$. The scan was a whole-brain acquisition, with 40 slices. A T1-weighted high-resolution structural scan was acquired after the functional scans $(\mathrm{TR}=12 \mathrm{msec}, \mathrm{TE}=5.6 \mathrm{msec}, 1 \times 1 \times 1 \mathrm{~mm}$ resolution). Ear plugs were used for noise reduction; foam padding was used to secure the head in the scanner and minimize head movements. Stimuli were projected to the back screen; a mirror was attached to the head coil and adjusted for the participants to see full screen. All fMRI preprocessing and analysis were performed using SPM12. To achieve T1 equilibrium, the first six dummy volumes were discarded. During preprocessing, we used the new segment (with six tissue classes) to optimize normalization. Otherwise, we used all default settings, and we performed slice timing correction. No participant had any abrupt motion change over $4 \mathrm{~mm}$. 


\section{MEG Recording and Preprocessing}

Recordings were made using a 275-channel CTF MEG system with superconducting quantum interference device-based axial gradiometers (VSM Med-Tech) and second-order gradients in a magnetically shielded room. Neuromagnetic signals were digitized continuously at a sampling rate of $480 \mathrm{~Hz}$ and then bandpass filtered in the $0.1-120 \mathrm{~Hz}$ range. Head positioning coils were attached to nasion, left, and right auricular sites to provide anatomical coregistration to a template brain. Preprocessing and analysis of MEG data were done using Fieldtrip (Oostenveld, Fries, Maris, \& Schoffelen, 2011). Independent component analysis (ICA) was performed on the continuous data, leading to the identification of blink, saccade, and cardiac components, which were removed. MEG data were subsequently parsed into epochs starting $1000 \mathrm{msec}$ before and ending $4000 \mathrm{msec}$ after the onset of the change point.

\section{Experimental Design and Statistical Analysis}

Participants performed 120 routes, with one change event occurring in each route (number of trials per condition was 17 on average, range $=11-25$, depending on the different scenarios used for counterbalancing routes taken). Each route started from a previous goal and ended at the new goal object for that trial. We used repeated-measures ANOVAs to test for behavioral differences (accuracy) between conditions. We also calculated $d^{\prime}$ and criterion (signal detection theory measures) to quantify the bias to take a false shortcut toward instead of away from a goal (both false alarms calculated relative to correct shortcuts, which are hits). We recorded the RT to make the first choice after the 4 sec elapsed, but due to the 4-sec delay, we do not interpret this as a traditional decision-making RT. We excluded control (i.e., freeze) events from all subsequent analyses, as it was a control condition and participants had low accuracy (fMRI: $60 \pm$ 2.8\%; MEG: $74 \pm 2.9 \%$ correct). Posttest debriefing indicated that this was likely due to participants finding it confusing that, despite the screen freezing for $4 \mathrm{sec}$, there was no apparent change, and thus, they changed their route choice in case they had missed a change. Additionally, given the limit on trial numbers, we were not able to investigate differences between correct rejections of false shortcuts and those that were taken mistakenly.

To analyze the fMRI data, we constructed multiple models based on a priori predictions from previous work (Howard et al., 2014). We used a standard preprocessing pipeline in SPM. A priori ROIs were small volume corrected using anatomical masks (WFU Pick atlas; Maldjian, Laurienti, Kraft, \& Burdette, 2003; TzourioMazoyer et al., 2002) and a functional mask for the dorsomedial PFC (Kaplan, King, et al., 2017) was employed in follow-up exploratory analysis. For completeness, we also report all results at an uncorrected threshold of $p<.001$, with a minimum of five contiguous voxels (Howard et al., 2014). This is provided to allow comparison with past data sets rather than to draw specific inferences about predicted responses. Note that we used all trials for an event type, irrespective of whether or not the participant was correct for not. General linear models were constructed: (1) Categorical effect of condition: The onset of the regressor was set at the onset of each change point separately (detour, shortcut, etc.) with a duration of $4 \mathrm{sec}$ (the duration of the change event). (2) Parametric effect of path distance: Same as above, but all the conditions were combined into one regressor (i.e., all change points combined, no differentiation), with the new path distance (after the change point) added as a parametric modulator (comprising a delta function at the change point that is parametrically modulated and then convolved with the hemodynamic response function). For these parametric regressors, we calculated the spatial parameters as in Howard et al. (2014) and Javadi et al. (2017). In brief, path distance, Euclidian distance, egocentric goal direction, and the number of optimal upcoming turns were calculated at each change point. All parameters were highly correlated ( $p<.001$; see Table 1$)$, except for path distance/ Euclidian distance and egocentric goal direction. Based on our previous work (Patai et al., 2019; Howard et al., 2014), our main analysis involved using path distance as an independent parametric regressor. We also considered a control model that included both path distance and egocentric goal direction, as these measures were not correlated. The other parameters were not explored independently. Spatial parameter values were rescaled between 0 and 1 , where 1 is the maximum value, for example, the greatest distance, calculated overall routes within participant. Finally, (3) Categorical effect of navigate versus follow: The onset of the start of the trial with a duration of $0 \mathrm{sec}$. The follow "trials" were just a few computer-directed steps (between 5 and 6) added to the start of a subset of the test trials (on average $37.5 \%$ ).

Table 1. Correlation between Spatial Parameters at Change Point/Start (Object Onset)

\begin{tabular}{lcccl}
\hline & $P D \%$ & $E D$ & \multicolumn{1}{c}{$E G D$} & \multicolumn{1}{c}{ Turns } \\
\hline PD & $0.43 * *$ & $0.64 * * 0.07 * *$ & $-0.03 /-0.05^{*}$ & $0.58 * * / 0.56 * *$ \\
PD\% & & $-0.1 * *$ & $0.09 * *$ & $0.49 * *$ \\
ED & & & $-0.02 /-0.07 * *$ & $0.11^{* *} /-0.11^{* *}$ \\
EGD & & & & $-0.22 * * / 0.04$ \\
\hline
\end{tabular}

PD\% does not exist at the start of trial, that is, when the target object is presented, as this measure assumes a change from the original path, which is only available at change points. Shown are $r$ values, with significance indicated by ${ }^{*} p<.001$, ${ }^{*} p<$ $.05 . \mathrm{PD}=$ new path distance after the change/original path distance at the onset; $\mathrm{PD} \%=$ relative change in path distance (compared with prechange path distance); EGD = egocentric goal direction; ED = Euclidian distance; Turns = number of upcoming turns. 
Table 2. Behavioral Summary: Mean ( $\pm S E M)$

\begin{tabular}{lcccccc}
\hline & Detour $(+8)$ & Detour $(+4)$ & Shortcut $(-8)$ & Shortcut $(-4)$ & False Shortcuts Toward & False Shortcuts Away \\
\hline fMRI accuracy (\%) & $64.1( \pm 3.9)$ & $80( \pm 2.3)$ & $84.5( \pm 2.5)$ & $84.1( \pm 2.8)$ & $65.8( \pm 3.1)$ & $81.3( \pm 1.9)$ \\
MEG accuracy $(\%)$ & $70.2( \pm 4.5)$ & $82.2( \pm 2.3)$ & $84.8( \pm 3.4)$ & $84.2( \pm 2.8)$ & $71.1( \pm 4.2)$ & $87.1( \pm 3.1)$ \\
\hline
\end{tabular}

To analyze the MEG data, we focused on event-related fields, as well as time-frequency analysis. Given the exploratory nature of this MEG study, we investigated effects of change type using all sensors and all time points the whole 4-sec change period. Here, we report significant effects found, cluster-corrected for multiple comparisons. For time-frequency analyses, we used the same exploratory method, but specifying the frequency ranges based on a priori bands as previously reported in the literature (3-7 Hz for theta [Kaplan, Bush, et al., 2017; Jacobs, 2014], 8-12 Hz for alpha, and 15-25 Hz for beta). Note that, for the theta band, we also confirmed this frequency band by measuring peak activity during an orthogonal period (power at the start of the trial comparing goal objects to follow arrows and found the group peak was at $5.2[ \pm 0.4 S E M] \mathrm{Hz})$. We also combined both lengths of shortcut $(-4 /-8)$ for comparison with false shortcuts.

Because of the nature of the task (free viewing during navigation) and despite the ICA correction during preprocessing, we were unable to exclude fully the possibility that some oscillatory signatures would be contaminated by eye movements. We therefore looked at the difference between the saccade variance as measured by ICA across different conditions and report these along with the event-related field and time-frequency results for completeness. We did not perform source localization on our MEG data set as we did not have any structural MRIs for realignment and no detailed head shape model available.

\section{RESULTS}

\section{Behavior}

Our primary measure of navigation was the accuracy of the whole route-in other words, whether participants took the optimal path to the target. We conducted a $2 \times 2$ repeated-measures ANOVA to test specifically the effect of Change type (detour vs. shortcut) and Magnitude (4/8 steps) of change and found a significant effect of Change type in both fMRI and MEG (fMRI: $F(1,20)=$ 35.03, $p<.001$; MEG: $F(1,23)=13.04, p<.001)$, a significant effect of Magnitude in the fMRI task only (fMRI: $F(1,20)=9.77, p=.005$; MEG: $F(1,23)=3.61, p=.07)$, and a significant interaction in both fMRI and MEG (fMRI: $F(1,20)=8.15, p=.01$; MEG: $F(1,23)=8.87, p=.007)$. We also conducted repeated-measures ANOVA to test for effects of all Terrain change type (including false shortcuts) on participants' accuracy in finding the correct path (Table 2 and Figure 3). We again found that there was a significant effect of Terrain change type (fMRI: $F(1$, $100)=14.7, p<.001 ;$ MEG: $F(1,115)=9.2, p<.001)$, such that detours $(+8)$ and false shortcuts toward the goal resulted in less optimal path taking (fMRI: both $t(1,20)<-3.6, p<.002$; MEG: $t(1,20)<-3.02, p<$ .006) compared with all other conditions (see Table 3 for comprehensive $t$ tests, all significant effects reported survive Bonferroni correction). There was also a significantly higher propensity (i.e., lower criterion) to take false shortcuts toward the goal, compared with false shortcuts away from the goal (fMRI: $t(1,20)=-4.71$, $p<.001$; MEG: $t(1,23)=-7.01, p<.001)$, as compared with real shortcuts.

\section{fMRI Results}

fMRI analyses revealed that bilateral hippocampus, bilateral parahippocampal cortex, retrosplenial cortex, as well as medial frontal areas were more active when participants were actively navigating than when they followed an arrow on the screen (Appendix A). Both the left and right hippocampus were significantly more active in the navigate than the follow condition (small-volume correction $p<.05$ ), in line with previous findings (Patai et al., 2019; Howard et al., 2014; for an overview, see Spiers \& Gilbert, 2015).

To match the behavioral data, we conducted a fixedeffects $2 \times 2$ model of change type by magnitude ( \pm 4 or 8 units) at the time of the change and found that there was a significant effect of change type, with detours showing increased visual cortex and medial frontal activity compared with shortcuts (family-wise error corrected

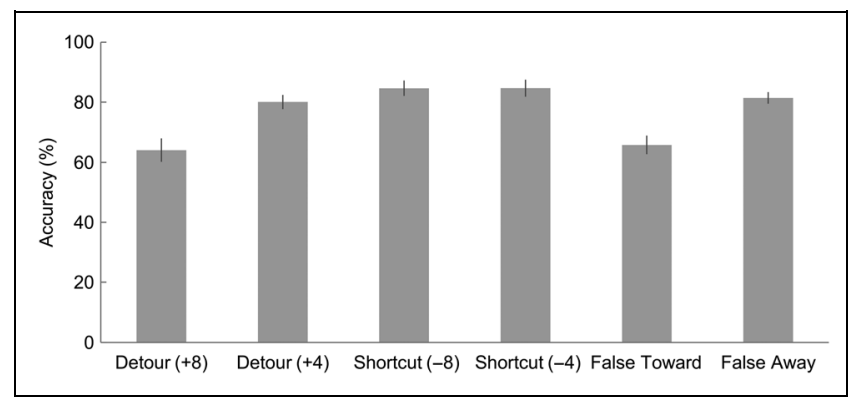

Figure 3. Accuracy across change types for the fMRI data set. See also Table 2. 
Table 3. Paired Samples $t$ Test Comparing All Terrain Change Types: Accuracy

\begin{tabular}{|c|c|c|c|c|c|c|}
\hline & \multicolumn{3}{|c|}{$f M R I$} & \multicolumn{3}{|c|}{$M E G$} \\
\hline & $t$ & $d f$ & $p$ & $t$ & $d f$ & $p$ \\
\hline Detour $(+8)$-Detour $(+4)$ & -4.21 & 20 & $<.001$ & -3.023 & 23 & .006 \\
\hline Detour $(+8)-$ Shortcut $(-8)$ & -5.659 & 20 & $<.001$ & -3.92 & 23 & $<.001$ \\
\hline Detour $(+8)-$ Shortcut $(-4)$ & -5.858 & 20 & $<.001$ & -3.483 & 23 & .002 \\
\hline Detour $(+8)-$ False shortcut away & -4.507 & 20 & $<.001$ & -4.809 & 23 & $<.001$ \\
\hline Detour $(+8)-$ False shortcut toward & -0.442 & 20 & .663 & -0.242 & 23 & .811 \\
\hline Detour $(+4)-$ Shortcut $(-8)$ & -1.468 & 20 & .158 & -0.728 & 23 & .474 \\
\hline Detour $(+4)-$ Shortcut $(-4)$ & -1.375 & 20 & .184 & -0.836 & 23 & .412 \\
\hline Detour $(+4)-$ False shortcut away & -0.487 & 20 & .632 & -2 & 23 & .057 \\
\hline Detour $(+4)-$ False shortcut toward & 3.655 & 20 & .002 & 3.07 & 23 & .005 \\
\hline Shortcut $(-8)-$ Shortcut $(-4)$ & -0.012 & 20 & .99 & 0.174 & 23 & .863 \\
\hline Shortcut $(-8)-$ False shortcut away & 1.047 & 20 & .307 & -0.649 & 23 & .523 \\
\hline Shortcut $(-8)-$ False shortcut toward & 5.059 & 20 & $<.001$ & 3.356 & 23 & .003 \\
\hline Shortcut $(-4)-$ False shortcut away & 1.068 & 20 & .298 & -1.127 & 23 & .271 \\
\hline Shortcut $(-4)-$ False shortcut toward & 6.197 & 20 & $<.001$ & 3.383 & 23 & .003 \\
\hline False shortcut away-False shortcut toward & 5.515 & 20 & $<.001$ & 6.041 & 23 & $<.001$ \\
\hline
\end{tabular}

at $p=.05$; detailed activations in Appendix A), no effect of magnitude and no interaction. Our main analyses were focused on predefined ROIs, including frontal areas as predicted by a model of navigation (Spiers \& Gilbert, 2015), and these are presented below.

\section{Frontal Activity in Response to Long Detours and False Shortcuts}

Next, we investigated frontal regions reported in previous studies comparing detours to nondetours: superior frontal gyrus, the right lateral prefrontal (rlPFC) and frontopolar cortex (Spiers \& Gilbert, 2015), using a combined mask of these areas. To examine whether detours would drive PFC activity more than shortcuts, we constructed a linear contrast of terrain change type (weighted $-2-11$ 2 with the order: detours $(+8)$, detour $(+4)$, shortcuts $(-4)$, shortcuts $(-8)$ ), which revealed a significant effect, with specifically the superior frontal gyrus and rlPFC activity scaling with the deviation from the optimal path before the change (Figure 4A and Appendix A). This effect appeared to be driven predominately by long detours $(+8)$, as comparing this condition directly to both shortcuts resulted in a significant effect in the combined frontal mask ( $p=.016, z=4.39$; for a full list of activations, see Appendix A), while this comparison was not significant for short detours $(+4)$.

False shortcuts toward the goal also significantly activated the rlPFC compared with shortcuts (Figure $4 \mathrm{~B}$ and Appendix A).
Additionally, when participants correctly rejected the false shortcut, there was more frontal activity (Table 5) versus more visual cortical and posterior cingulate activity when they chose incorrectly (Appendix A). By contrast, false shortcuts away from the goal did not drive activity in rlPFC.

We also found that, during processing of detours and false shortcuts, there was an increase in dorsomedial cortex (dorsal ACC [dACC]), an area previously reported during decision-making in a spatial context (Kaplan, King, et al., 2017), and we report this effect and those in the combined frontal mask, as well as the individual areas, in Table 5. Importantly, long detours also significantly activated the dACC compared with short detours $(p=.033$, $z=4.11$; for a full list of activations, see Appendix A), underscoring that these prefrontal effects are not driven by purely visual changes in the lava movement.

\section{Caudate, but Not Hippocampal, Activity Responds to Changes in the Path Distance to the Goal at Detours}

Based on our previous findings (Howard et al., 2014), we predicted that hippocampal activity would track the change in the path distance to the goal when the structure of the environment changed. We found no evidence to support this prediction, even with specific ROIs and at a low uncorrected threshold $(p<.005)$. This was also true when large detours $(+8)$ were directly compared with small detours $(+4)$. By contrast, we found that activity in the caudate nucleus bilaterally tracked the change in the path distance across all types of events (see 


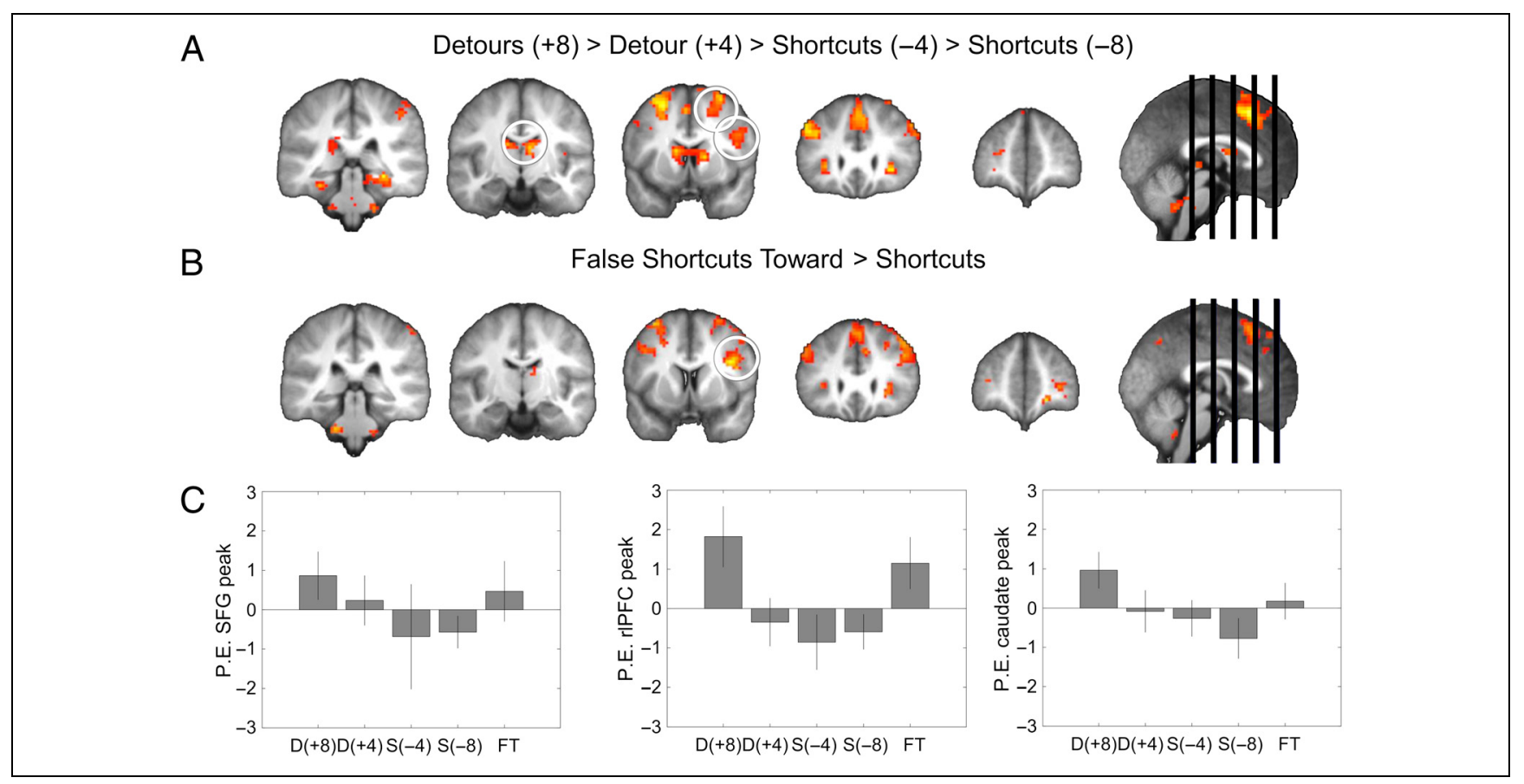

Figure 4. Prefrontal areas involved during processing of terrain changes. (A) Superior frontal gyrus (SFG), right lateral prefrontal cortex (rlPFC), and bilateral caudate were engaged in the linear contrast of detours and shortcuts (detours $(+8)>$ detour $(+4)>$ shortcuts $(-4)>$ shortcuts $(-8)$ ) and (B) rlPFC when comparing false shortcuts toward the goal to shortcuts. Figures are thresholded at $p=.005$ uncorrected. (C) Parameter estimates from the peak voxels in the contrast from A and B, for illustration purposes only. P.E. = parameter estimate.

Table 5), complementing past evidence that this region tracked the magnitude of change in the path at detours (Howard et al., 2014).

\section{The Hippocampus Does Not Selectively Code for Total Path Distance at Detours}

Given previous findings that the hippocampus codes for distance during navigation, we predicted a parametric modulation of hippocampal activity with new path distance at terrain change points, specifically at detours (Howard et al., 2014). Although we found a significant effect in the right hippocampus when combining all terrain change points, this effect was not specific to the hippocampus and was not present when looking at detours and shortcuts separately. Therefore, we cannot conclude that the hippocampus has a specific role in coding distance at points during a route that require a plan update, such as detours. However, given the experimental design, it was not possible to have new path distances at terrain change points that were orthogonal to the type of change, in other words the parametric effect of new path distance was confounded by terrain change type (e.g., detours $(+8)$ resulted in longer total new path distances than detours $(+4)$ ). In the future, it will be important to dissociate the magnitude of the change from the resulting overall upcoming distance to directly test the effect of hippocampal distance coding at detours.

\section{Path Visibility during False Shortcuts}

Because our maze environment was in an open plane participants could see ahead (see Figure 1), it is possible that paths could have been selected using a purely visual search of the available paths to the remembered hidden goal location (as opposed to relying on memory for the layout of the environment). This is particularly relevant for false shortcuts where the path needs to be rejected for optimal behavior. Peak activity in the rlPFC activity seen in the contrast of false shortcut toward versus shortcuts (Figure 4B) was not significantly different between "visible" and "not clearly visible" false shortcuts toward the goal $(p>1)$. Thus, rlPFC activity was not purely driven by the visible paths. Nonetheless, we should be

Table 4. Accuracy (\% Errorless Trials) as a Function of Visibility (fMRI Data Set)

\begin{tabular}{lcccccc}
\hline & Detour $(+8)$ & Detour $(+4)$ & Shortcut $(-8)$ & Shortcut $(-4)$ & False Shortcuts Toward & False Shortcuts Away \\
\hline Visible & $69.2( \pm 7.5)$ & $81.7( \pm 2.0)$ & n/a & n/a & $75.9( \pm 3.9)$ & $82.6( \pm 2.0)$ \\
Not clearly visible & $64.8( \pm 3.8)$ & $74.2( \pm 6.0)$ & $84.5( \pm 2.5)$ & $84.1( \pm 2.8)$ & $52.8( \pm 4.6)$ & $77.7( \pm 6.3)$ \\
\hline
\end{tabular}


Table 5. Results of Small-volume Correction in ROIs during Terrain Changes

\begin{tabular}{|c|c|c|c|}
\hline & $D+8>D+4>S-4>S-8$ & $\begin{array}{c}\text { False Shortcut } \\
\text { Toward }>\text { Shortcuts }\end{array}$ & $\begin{array}{c}\text { False Shortcut Toward } \\
\text { Correct > Incorrect }\end{array}$ \\
\hline Combined frontal mask & $p=.001, Z=4.97$ & $p=.001, Z=5.28$ & $p<.001, Z=2.80$ \\
\hline Superior frontal gyrus & $p=.001, Z=4.97$ & $n s$ & $p<.001, Z=2.80$ \\
\hline Frontopolar & $n s$ & $n s$ & ns \\
\hline rlPFC & $p=.006, Z=4.26$ & $p=.001, Z=5.28$ & $p=.014, Z=4.08$ \\
\hline dACC & $p=.002, Z=4.73$ & $p=.023, Z=4.20$ & $p<.001, Z=2.80$ \\
\hline Caudate & $p=.02, Z=3.93$ & ns & ns \\
\hline Left HC & ns & ns & ns \\
\hline Right HC & $n s$ & $n s$ & ns \\
\hline
\end{tabular}

All results reported are significant after false discovery rate correction for multiple comparisons. $\mathrm{HC}=$ hippocampus; rlPFC $=$ right-lateral prefrontal cortex; dACC $=$ dorsal anterior-cingulate cortex.

cautious of interpreting this as evidence that PFC was operating on the memory of the paths to solve the task. Indeed, as would be expected, when the path was visible, participants were significantly more accurate (percentage of errorless paths when visible: $76 \% \pm 4 \%$, percentage of errorless paths when the path was not clearly visible: $53 \% \pm 5 \%$ ). Because of trial numbers, we cannot directly compare PFC activity in visible versus nonvisible trials as a function of performance.

To explore this further, we examined whether visibility impacted the number of errors in the other conditions and found that there was an effect of condition, $F(1,57)=7.45, p<.001$, and visibility, $F(1,19)=9.69$, $p=.006$, but no interaction $(p>.1$; see Table 4$)$. Only the false shortcuts toward the goal showed a significant effect of visibility on paired $t$ tests, $t(1,21)=4.2, p<$ .001 (all others $p>.2$; Table 4 ). There were no shortcuts where the path from the current location to the goal was entirely visible at the time point when the shortcut occurred, hence the "n/a" in Table 4.

\section{Electrophysiological Indices of Navigation}

\section{Event-related Fields}

A repeated-measures ANOVA revealed a significant effect of Change type and Magnitude but no interaction. Two significant time periods emerged from our analysis of Change type: 400-600 msec and 700-1000 msec after the onset of the terrain change. In both of these, there was a larger deflection for shortcuts than detours: an earlier left frontotemporal effect, followed by a right temporal-occipital effect. However, the saccade variance was significantly different between conditions (paired $t$ test, $t(1,23)=2.5$, $p=.02$ ) and responses driven by detours versus shortcuts may relate to the visual differences of lava disappearing or

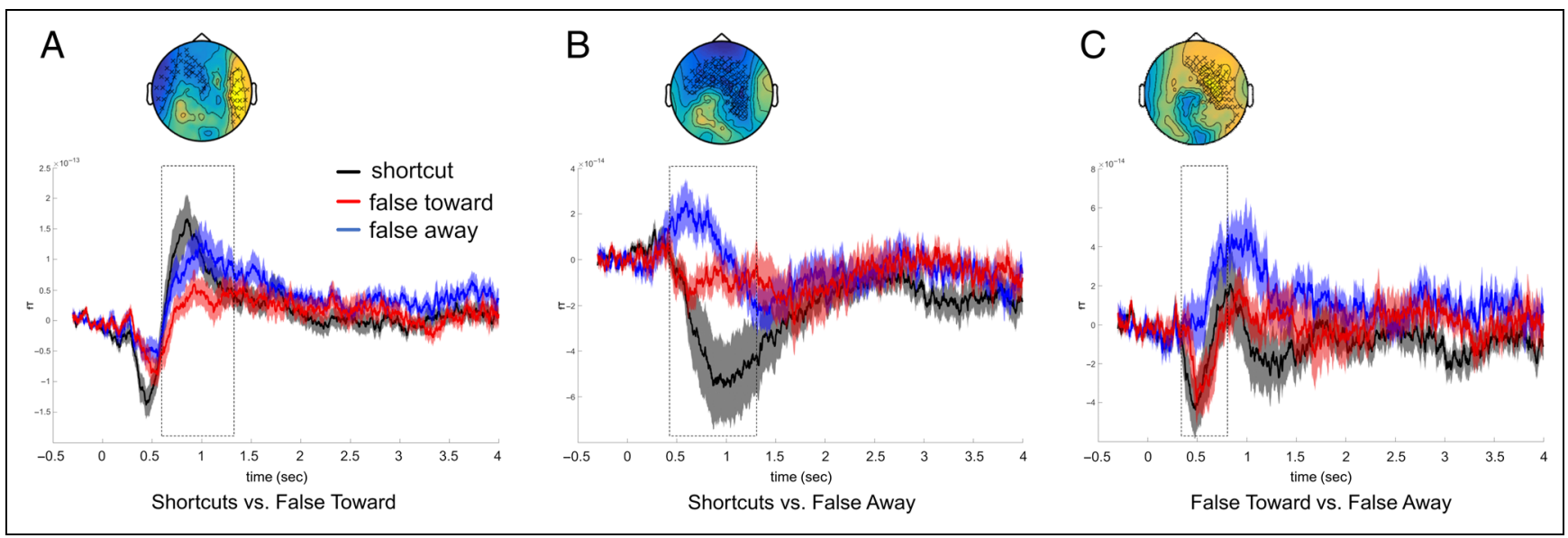

Figure 5. Event-related field changes to shortcuts and false shortcuts. We found significant differences in the event-related fields between (A) shortcuts versus false toward, positive cluster 180-540 msec, and negative cluster $p=.014$, time: 660-1000 msec; (B) shortcuts versus false away 540-1340 msec; and (C) false shortcuts toward versus away from the goal between 460 and $880 \mathrm{msec}$, after the onset of the change point (opening in the lava). Displayed in each panel is the topography of the difference between the conditions with the significant sensors marked by xs. The plotted event-related field is the average $( \pm S E M)$ over the significant sensors, with the significant time period highlighted with dashed boxes. Note that data from the detours are not presented due to potential eye movement confounds. $\mathrm{fT}=$ femtoTesla. 
being added. To investigate neural responses related to the magnitude of the change in path instigated by the terrain change point, we combined long detours and shortcuts $( \pm 8)$ and short detours and shortcuts $( \pm 4)$. We found a significant Frontal effect from 250 to $800 \mathrm{msec}$, with changes that induced a large change in path showing a larger deflection. However, because of eye movements, we cannot explicitly exclude the possibility that effects are contaminated by differences in saccadic behavior between conditions (as measured by variance in the saccade components derived from the ICA): There was a significant effect of Eye movements between conditions (type, $F(1,23)=6.4, p<$ .019 ; magnitude, $F(1,23)=24.02, p<.001$; interaction, $F(1,23)=64.04, p<.001)$, with detours $(+8)$ showing the largest variance compared with all conditions (all $t(1$, 23) $>4.1, p<.001)$. Thus, we focus our analysis on shortcuts and false shortcuts, which were not significantly different on eye movement variance (shortcuts vs. false shortcut toward, $t(1,23)<1.5, p>.1$; shortcuts vs. false shortcut away, $t(1,23)=1.5, p=.07$; false shortcuts toward vs. away, $t(1,23)=0.26, p>.1)$.

\section{Early Differentiation of Shortcuts from False Shortcuts}

Comparison of shortcuts and false shortcuts (both toward and away) allowed us to investigate how the brain responds to changes in the environment that result in different benefits of outcome, that is, real shortcuts are useful and lead to the goal via shorter path. False shortcuts should be processed differently from shortcuts if participants have an accurate understanding of the layout of the maze and memory of the goal locations. When examining the wait period after a change point (a 4-sec delay after the change in lava during which participants had to decide which route to take), we found that shortcuts had significantly different event-related fields from both types of false shortcut (shortcuts vs. false toward: negative cluster $p=.045$, time: $660-1340 \mathrm{msec}$, sensor distribution: bilateral temporal-frontal, Figure $5 \mathrm{~A}$; shortcuts vs. false away: negative cluster $p=.003$, time: $540-1340 \mathrm{msec}$, sensor distribution: right frontal-temporal, Figure 5B). Moreover, false shortcuts toward the goal were different from false shortcuts away from the goal (negative cluster $p=.034$, time: $460-880 \mathrm{msec}$, sensor distribution: right frontal-temporal, Figure 5C). To investigate early feedbackrelated negativity that has been typically associated with reward processing and has specifically been linked to signed reward prediction error in reinforcement learning paradigms (Holroyd \& Coles, 2002), we also focused on the early part of the trial $(0-1000 \mathrm{msec})$ and found a significant difference between shortcuts and false shortcuts toward the goal (negative cluster, $p=.04,180-540 \mathrm{msec}$ ) on central posterior sensors. Thus, rapidly after a terrain changes (as early as $180 \mathrm{msec}$ ) neural processing distinguishes between potential useful new paths from those that will be detrimental in reaching the goal and after around half a second distinguishes two different types of false shortcut.

\section{Oscillatory Markers during Navigational Choices}

Prior research has indicated that oscillations at theta frequencies (3-7 Hz in humans) are involved in navigation and spatial memory (Eschmann, Bader, \& Mecklinger, 2018; Bohbot, Copara, Gotman, \& Ekstrom, 2017; Hasselmo, Hinman, Dannenberg, \& Stern, 2017; Namboodiri, Levy, Mihalas, Sims, \& Hussain Shuler, 2016; Chakravarthy \& Balasubramani, 2015; Hartley, Lever, Burgess, \& O'Keefe,
Figure 6. Distinct timefrequency markers for processing different types of shortcuts. We found increased activity in the theta band when comparing shortcuts to both types of false shortcut (toward: 50-2140 msec; away: 0-3160 msec) as well as a trend toward increased theta for false shortcuts toward compared away from the goal (1450-3000 msec). Note that data from the detours are not presented due to potential eye movement confounds.

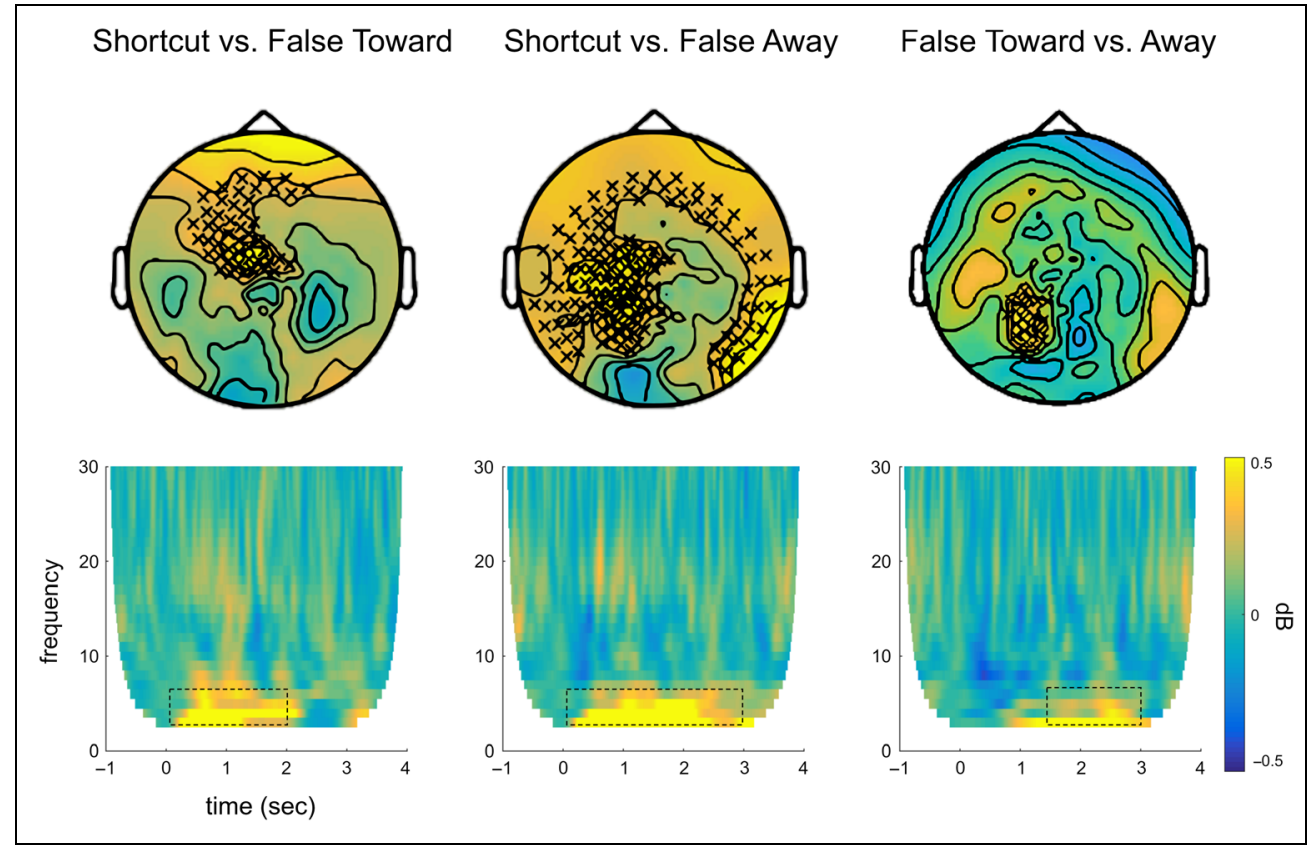


2014; Jocham et al., 2014; Kaplan et al., 2012, 2014; Snider, Plank, Lynch, Halgren, \& Poizner, 2013; Cornwell et al., 2008; Buzsáki, 2005; Hasselmo, Hay, Ilyn, \& Gorchetchnikov, 2002). We therefore examined this frequency band (3-7 Hz; see Methods for details). Shortcuts led to significantly increased theta power, compared with both types of false shortcut for nearly the whole duration of the epoch ( $4 \mathrm{sec}$ ) after the change point (shortcut vs. false shortcut toward: positive cluster $p=.037$, 50-2140 msec with a frontal-central distribution; shortcut vs. false away: positive cluster $p<.001,0-3160 \mathrm{msec}$ with a bilateral frontal-temporal distribution; see Figure 6). We also found a trend for increased theta power for false shortcuts toward the goal compared with away from the goal, starting late after the change point over left parietal sensors (positive cluster: 1450-3000 msec, $p=.09$; Figure 6). Other frequency bands did not reveal any effects between shortcuts and false shortcuts, neither in the alpha band (12-18 Hz) or in the beta band (15$25 \mathrm{~Hz}$ ), except for the contrast of shortcuts compared with false shortcuts toward, where shortcuts exhibited significantly more beta power (positive cluster $p=$ $.005,640-1430 \mathrm{msec}$ with bilateral temporal and occipital distribution). Additionally, there was no strong relationship between behavioral accuracy and the theta response differences (shortcut vs. false shortcut toward $r=.34, p=.099$; shortcut vs. false shortcut away $r=.19$, $p=.37$; false shortcuts toward vs. away $r=.36, p=$ $.085)$, indicating the changes in theta response were not a simple function of behavioral choice or difficulty.

\section{DISCUSSION}

A core tenet of the cognitive map theory is that internal representations support flexible navigation, enabling an animal to make use of shortcuts and take efficient detours (O'Keefe \& Nadel, 1978; Tolman, 1948). Despite the wide acclaim for this idea, little research, especially in humans, has been directed at understanding the neural mechanisms, which underlie such adaptive behavior (for reviews, see Epstein et al., 2017; Spiers \& Gilbert, 2015). Using fMRI and MEG and a VR task involving navigation through a landscape that changed layout sporadically, we examined the neural responses to forced detours, novel shortcuts, and false shortcuts. We found (i) superior and lateral PFC and caudate activity was evoked by detours, maximally when there was a large change in the path, (ii) rlPFC responded when false shortcuts to the goal needed to be avoided and (iii) evoked and induced neural activity differentiated false shortcuts from real shortcuts as early as $180 \mathrm{msec}$.

\section{The Role of PFC, Hippocampus, and Caudate in Responding to Detours and Shortcuts}

Based primarily on evidence from nine fMRI studies, Spiers and Gilbert (2015) provided preliminary predictions about how PFC and the hippocampus might respond to forced detours and changes in the layout of an environment. Lateral PFC was suggested to provide a prediction error signal in response to changes in the path options (responding whenever an unpredicted change in the possible paths occurs). The superior and anterior PFC was speculated to support reformulation of the route plan (responding at all events that require reconsidering the change in route plan). The hippocampus was postulated to simulate the future path the goal (responding the greater the increase in the path to the goal), drawing on rodent place cell studies (Ólafsdóttir, Barry, Saleem, Hassabis, \& Spiers, 2015; Pfeiffer \& Foster, 2013). Here, we failed to find evidence that the hippocampus specifically encodes the change in the path distance to the future goal. One possibility is that the hippocampus simulates future possible scenes (Hassabis \& Maguire, 2007), reconstructing the different locations that lie between the current location and the future goal (Javadi et al., 2017; Spiers \& Barry, 2015). In the case of the current study, the environment was sparse with few features to distinguish different parts of the island, which might explain why we did not observe a correlation between the hippocampus and the change in path to the goal. Notably, previous studies reporting hippocampal activity correlated with the future path to the goal used real-world stimuli with nameable landmarks located along the paths (Patai et al., 2019; Javadi et al., 2017; Howard et al., 2014).

By contrast to the hippocampus, we found that activity in lateral and superior PFC, as well as the caudate, responded maximally when there was a large change in the path to the goal and not during false shortcuts, which also involve inhibition but no need to update the path. The caudate response is consistent with a prior result from Howard et al. (2014), who found that the larger the distance induced by the detour, the more activity was elicited in the caudate nucleus. Thus, speculatively the caudate activity may relate to a signal linked to updating the transition structure in the environment at that particular location where the change occurs, consistent with this region coding a prediction error about future events (O'Doherty et al., 2004). Consistent with our caudate responses reflecting a model-based updating process, a previous fMRI study of navigation in a continually changing environment found that caudate activity correlated with parameters of a model-based representation of the environment (Simon \& Daw, 2011; Dickinson \& Balleine, 2002). More recently, caudate activity has been shown for events where pauses were implemented, as well as when there were unexpected changes in distance (RibasFernandes, Shahnazian, Holroyd, \& Botvinick, 2019).

The PFC responses we observed are in agreement with the predicted roles of the superior PFC supporting resolving path conflict and the rlPFC processing a prediction error signal between the predicted state of the world and the encountered layout (Spiers \& Gilbert, 
2015). Two types of prediction error could be processed in the current paradigm. One is the signed prediction error signal linked to the difference in the path before and after the change in the layout (+ve for detours, -ve for shortcuts). The other is an unsigned prediction error where the amount of change is coded rather than the direction of change (+ve for both detours and shortcuts). Our results show a wide network of regions, including our PFC and caudate ROIs, was driven in a manner consistent with the signed prediction error (maximal for +8 detours). Our results thus align more strongly with models in which PFC and caudate code the increase in path, and rather than being driven in a clear linear manner by the signed prediction error, the data suggest these regions might be driven in a threshold manner by large detours over the other conditions. Future research carefully varying along a broader range the amount of path change at detours will be required to explore these possibilities.

It is possible PFC responses to detours are driven by the presence of the physical barrier appearing to block the route. This is certainly a possibility in several past studies (e.g., Iaria et al., 2008; Maguire et al., 1998), though not all (see Howard et al., 2014) However, because rlPFC was more active for false shortcuts compared with shortcuts and these two events are visually similar (one unit of lava is removed to create a new path), it seems more likely rlPFC is driven by planning demands rather than the visual processing of a barrier. This response is consistent with it playing a role in behavioral control: suppressing the prepotent response to move toward the goal drawing on the observation that there is now a barrier or that there is a new opening that is not helpful (Spiers \& Gilbert, 2015).

\section{The Role of Temporal and Oscillatory Dynamics during the Differentiation of Useful versus Deceptive Shortcuts}

We observed increased theta power when participants considered shortcuts and false shortcuts toward the goal. As false shortcuts away can be distinguished on the basis of the goal direction whereas shortcuts and false shortcuts toward predominately required memory for the structure of the environment to distinguish them, the increased theta power is consistent with arguments that theta aids future navigational planning through retrieval (Kaplan et al., 2014) and imagery (Kaplan, Bush, et al., 2017). Additionally, increased theta activity was elicited when a longer distance was expected in the future than shorter one (Bush et al., 2017; Vass et al., 2016; Caplan, Madsen, Raghavachari, \& Kahana, 2001), in which case, we would have expected increased theta synchrony for false shortcuts in our data if participants were engaging in simulating a future path (which would be longer if they took a false shortcut-unless the accuracy of the simulation drives the theta effect). However, the increased theta at shortcuts we found may be related to reward processing (for a review, see Cavanagh \& Frank, 2014), as participants anticipate a shortened path to their goal. Alternatively, the theta response may be more consistent with an increase in the conflict between choices or stimuli, as has been observed in previous navigation studies (Watrous, Fried, \& Ekstrom, 2011; Weidemann, Mollison, \& Kahana, 2009).

The increased beta power found when contrasting shortcuts to false shortcuts toward the goal could be related to earlier movement preparation during shortcuts, if participants are more certain of their choice, or it may be related to recall processes, which have been found to involve beta power desynchronization (Hanslmayr, Staresina, \& Bowman, 2016; Hanslmayr, Staudigl, \& Fellner, 2012). Thus, less beta power in false shortcuts toward the goal may be indicative of increased mnemonic processing of the spatial layout of the maze, and given the bilateral posterior distribution, it may specifically be related to recalling visual layouts to aid path choice, similarly to context reinstatement during recall (Staudigl, Vollmar, Noachtar, \& Hanslmayr, 2015). Though beta desynchronization has been found with concurrent theta synchronization (Hanslmayr et al., 2012, 2016), our data indicate that these two neural signatures may be underlying different cognitive processes while making a decision during spatial navigation. Future studies should aim at dissociating the immediate reward of a shorter path (as in the case of shortcuts) from the overall reward associated with reaching a particular goal to dissociate oscillatory changes related to decisionmaking and spatial memory.

\section{Relationship between fMRI and MEG Activations during Navigational Choices}

Our study provides an opportunity to examine the convergence of neural activations found using different methodologies. Because of methodological issues we were not able to apply source reconstruction in the MEG data to directly compare sources in MEG with localized BOLD signal in the fMRI study. Nonetheless, the integration of the methods does allow us to align the findings to support certain models of how the navigation system may operate. The medial frontal activity in the dACC that we report during detours and false shortcuts toward as each compared with shortcuts has been shown to be related to feedback-related negativity (Hauser et al., 2014), an electrophysiological index of reward prediction errors. The feedback-related negativity commonly shows a larger negative deflection at mid-central sites for punishment compared with reward (Holroyd \& Coles, 2002), which we replicated in our MEG data set (early more negative deflection for false shortcuts toward the goal), and additionally, we found that the dACC was most active for long detours $(+8)$, which add the most delay before reaching the rewarded target in our experiment. 
Thus, it is plausible that the dACC gives rise to the rapid response elicited when the path to the future rewarded target requires more extensive reevaluation. Future work will be required to validate this speculation.

We found increased frontal BOLD activity during false shortcuts toward the goal compared with shortcuts, while theta power was increased for shortcuts over frontal-central sensors. Conversely, posterior beta power increases for shortcuts compared with false shortcuts toward the goal were paralleled by BOLD increases in visual areas (see Appendix A). These results agree with previous reports of an inverse relationship between lowfrequency oscillatory power and BOLD activity (Conner, Ellmore, Pieters, DiSano, \& Tandon, 2011; Scheeringa et al., 2009), but beta is usually also inversely related while gamma frequencies show a positive relationship with BOLD (Conner et al., 2011; Scheeringa et al., 2011). Thus, future research will be needed to elucidate whether the relationship between oscillatory and BOLD activity is altered for different brain regions, which would be better explored using simultaneous fMRI and EEG, and second to overcome methodological issues with recording electrophysiology during dynamic tasks with the consequent eye movement confounds.

\section{Conclusion}

In our study, we report changes in neural activity during flexible navigation using convergent evidence from functional imaging and magnetoencephalography. We find that during detours, when longer paths to the goal are required, and during false shortcuts, when plausible but unhelpful paths need to be rejected, there is an increase in superior-lateral and medial frontal areas. This is mirrored by changes in theta and beta band oscillations as well as early deflections in event-related fields, specifically between shortcuts and false shortcuts toward the goal. Future research should aim to dissociate reward, memory, and attentional processes during navigation and the learning process of the environment (e.g., use of maps) to better understand the neural dynamics of flexible goal-directed navigation.

\section{APPENDIX A}

\begin{tabular}{|c|c|c|c|c|c|c|c|}
\hline Contrast Name & Region Label & Extent & $t$ Value & $z$ Value & $x$ & $y$ & $z$ \\
\hline \multirow[t]{23}{*}{ Nav $>$ Follow } & $\mathrm{L}$ angular gyrus & 101 & 8.789 & 5.56 & -42 & -73 & 35 \\
\hline & L precuneus & 461 & 8.278 & 5.40 & -6 & -58 & 17 \\
\hline & L fusiform gyrus & 280 & 7.569 & 5.14 & -30 & -31 & -22 \\
\hline & $\mathrm{R}$ fusiform gyrus & 212 & 7.564 & 5.14 & 33 & -43 & -19 \\
\hline & L superior medial gyrus & 46 & 6.104 & 4.53 & -9 & 29 & 38 \\
\hline & L middle frontal gyrus & 85 & 5.732 & 4.36 & -24 & 17 & 53 \\
\hline & R cerebelum (VIII) & 157 & 5.656 & 4.32 & 33 & -70 & -43 \\
\hline & R superior orbital gyrus & 73 & 5.612 & 4.30 & 21 & 32 & -10 \\
\hline & Location not in atlas & 19 & 5.207 & 4.09 & 39 & -13 & 38 \\
\hline & Location not in atlas & 12 & 5.140 & 4.06 & -6 & -40 & 29 \\
\hline & Location not in atlas & 42 & 5.136 & 4.05 & 15 & -19 & -16 \\
\hline & $\mathrm{R}$ precentral gyrus & 26 & 4.722 & 3.83 & 36 & -22 & 50 \\
\hline & L middle frontal gyrus & 20 & 4.653 & 3.79 & -36 & 47 & 11 \\
\hline & L inferior temporal gyrus & 16 & 4.430 & 3.65 & -48 & -55 & -19 \\
\hline & L cerebelum (Crus 2) & 7 & 4.327 & 3.59 & -33 & -76 & -43 \\
\hline & Location not in atlas & 8 & 4.311 & 3.58 & 30 & -64 & -34 \\
\hline & Location not in atlas & 22 & 4.310 & 3.58 & -30 & 32 & -16 \\
\hline & Location not in atlas & 11 & 4.250 & 3.55 & -36 & -16 & 38 \\
\hline & L superior frontal gyrus & 12 & 4.174 & 3.50 & -27 & 59 & 11 \\
\hline & R cerebelum (VII) & 5 & 4.095 & 3.45 & 42 & -52 & -43 \\
\hline & L superior orbital gyrus & 7 & 4.071 & 3.43 & -12 & 56 & -1 \\
\hline & L medial temporal pole & 6 & 3.992 & 3.38 & -45 & 14 & -22 \\
\hline & R IFG (p. triangularis) & 9 & 3.901 & 3.32 & 45 & 29 & 20 \\
\hline
\end{tabular}


APPENDIX A (continued)

\begin{tabular}{|c|c|c|c|c|c|c|c|}
\hline Contrast Name & Region Label & Extent & $t$ Value & $z$ Value & $x$ & $y$ & $z$ \\
\hline \multirow[t]{26}{*}{ Detour $>$ Shortcut } & L middle occipital gyrus & 556 & 8.113 & 6.91 & -12 & -97 & 5 \\
\hline & $\mathrm{R}$ calcarine gyrus & 943 & 6.927 & 6.11 & 15 & -97 & 8 \\
\hline & L posterior-medial frontal & 162 & 6.142 & 5.54 & -6 & 17 & 50 \\
\hline & L middle frontal gyrus & 195 & 5.802 & 5.28 & -27 & 8 & 59 \\
\hline & L middle occipital gyrus & 74 & 5.735 & 5.23 & -21 & -67 & 41 \\
\hline & $\mathrm{R}$ middle frontal gyrus & 55 & 4.835 & 4.51 & 51 & 23 & 38 \\
\hline & L IFG (p. triangularis) & 58 & 4.636 & 4.35 & -45 & 29 & 32 \\
\hline & L cerebelum (VI) & 32 & 4.521 & 4.25 & -30 & -76 & -16 \\
\hline & L precuneus & 7 & 4.515 & 4.25 & -6 & -64 & 47 \\
\hline & $\mathrm{R}$ superior frontal gyrus & 84 & 4.289 & 4.06 & 24 & 5 & 59 \\
\hline & L fusiform gyrus & 9 & 4.113 & 3.91 & -33 & -34 & -19 \\
\hline & L cerebelum (IX) & 35 & 4.102 & 3.90 & -12 & -46 & -46 \\
\hline & R IFG (p. orbitalis) & 15 & 4.095 & 3.89 & 30 & 26 & -4 \\
\hline & Location not in atlas & 13 & 4.056 & 3.86 & 21 & -52 & 20 \\
\hline & L middle frontal gyrus & 17 & 4.046 & 3.85 & -33 & 56 & 8 \\
\hline & $\mathrm{R}$ thalamus & 26 & 4.013 & 3.82 & 12 & -22 & 17 \\
\hline & L IFG (p. orbitalis) & 10 & 3.971 & 3.78 & -33 & 29 & -4 \\
\hline & Location not in atlas & 9 & 3.935 & 3.75 & 21 & -37 & -40 \\
\hline & Location not in atlas & 6 & 3.882 & 3.70 & -21 & -37 & -43 \\
\hline & R superior medial gyrus & 16 & 3.863 & 3.69 & 6 & 35 & 59 \\
\hline & Location not in atlas & 8 & 3.684 & 3.53 & 6 & -28 & -1 \\
\hline & $\mathrm{R}$ fusiform gyrus & 5 & 3.648 & 3.50 & 33 & -10 & -31 \\
\hline & $\mathrm{R}$ middle frontal gyrus & 11 & 3.635 & 3.49 & 45 & 32 & 26 \\
\hline & L thalamus & 10 & 3.631 & 3.48 & -18 & -31 & 17 \\
\hline & Location not in atlas & 11 & 3.563 & 3.42 & 3 & 5 & 14 \\
\hline & L thalamus & 5 & 3.488 & 3.36 & -9 & -19 & 20 \\
\hline \multirow[t]{11}{*}{$\mathrm{D}(+8)>\mathrm{D}(+4)>\mathrm{S}(-4)>\mathrm{S}(-8)$} & L inferior occipital gyrus & 682 & 9.817 & 5.87 & -24 & -97 & 2 \\
\hline & $\mathrm{R}$ linual gyrus & 946 & 8.307 & 5.40 & 15 & -94 & 2 \\
\hline & L superior frontal gyrus & 186 & 7.115 & 4.97 & -21 & 17 & 65 \\
\hline & L superior medial gyrus & 180 & 6.549 & 4.73 & -6 & 20 & 47 \\
\hline & L middle occipital gyrus & 145 & 6.376 & 4.66 & -24 & -67 & 44 \\
\hline & L middle frontal gyrus & 59 & 6.227 & 4.59 & -48 & 29 & 35 \\
\hline & R calcarine gyrus & 31 & 6.044 & 4.51 & 21 & -55 & 14 \\
\hline & $\mathrm{R}$ thalamus & 46 & 5.715 & 4.35 & 12 & -19 & 17 \\
\hline & $\mathrm{R}$ middle frontal gyrus & 44 & 5.541 & 4.26 & 51 & 20 & 38 \\
\hline & Location not in atlas & 14 & 5.538 & 4.26 & 18 & -37 & -43 \\
\hline & $\mathrm{R}$ middle frontal gyrus & 131 & 5.486 & 4.24 & 30 & 5 & 62 \\
\hline
\end{tabular}




\begin{tabular}{|c|c|c|c|c|c|c|c|}
\hline Contrast Name & Region Label & Extent & $t$ Value & $z$ Value & $x$ & $y$ & $z$ \\
\hline & L cerebelum (VII) & 27 & 5.435 & 4.21 & -6 & -79 & -37 \\
\hline & Location not in atlas & 11 & 5.366 & 4.17 & 27 & -61 & -31 \\
\hline & R IFG (p. orbitalis) & 21 & 5.351 & 4.17 & 30 & 23 & -4 \\
\hline & $\mathrm{R}$ caudate nucleus & 104 & 4.901 & 3.93 & 15 & 8 & 8 \\
\hline & R cerebelum (IX) & 14 & 4.889 & 3.92 & 15 & -46 & -46 \\
\hline & L cerebelum (IX) & 46 & 4.832 & 3.89 & -12 & -46 & -46 \\
\hline & R superior frontal gyrus & 5 & 4.715 & 3.82 & 24 & 32 & 56 \\
\hline & L superior frontal gyrus & 44 & 4.585 & 3.75 & -30 & 59 & 5 \\
\hline & L insula lobe & 32 & 4.566 & 3.74 & -36 & 17 & -1 \\
\hline & Location not in atlas & 6 & 4.506 & 3.70 & -6 & -28 & -1 \\
\hline & L precentral gyrus & 7 & 4.499 & 3.70 & -39 & 2 & 38 \\
\hline & Location not in atlas & 7 & 4.333 & 3.60 & 6 & -25 & -1 \\
\hline & L cerebelum (VIII) & 10 & 4.330 & 3.59 & -30 & -70 & -52 \\
\hline & Location not in atlas & 7 & 4.270 & 3.56 & -9 & -43 & -34 \\
\hline & Location not in atlas & 6 & 3.984 & 3.38 & -3 & -49 & -40 \\
\hline & R IFG (p. opercularis) & 12 & 3.847 & 3.29 & 48 & 8 & 29 \\
\hline \multirow[t]{19}{*}{$\mathrm{D}(+8)<\mathrm{D}(+4)<\mathrm{S}(-4)<\mathrm{S}(-8)$} & $\mathrm{R}$ cuneus & 73 & 6.259 & 4.61 & 6 & -82 & 26 \\
\hline & L superior temporal gyrus & 24 & 5.759 & 4.37 & -45 & -40 & 26 \\
\hline & Location not in atlas & 56 & 5.721 & 4.35 & 45 & -28 & 29 \\
\hline & R PCC & 41 & 5.644 & 4.32 & 12 & -49 & 35 \\
\hline & R supramarginal gyrus & 46 & 5.532 & 4.26 & 57 & -52 & 29 \\
\hline & L middle temporal gyrus & 11 & 5.269 & 4.12 & -57 & -61 & 23 \\
\hline & Location not in atlas & 12 & 4.947 & 3.95 & -18 & -28 & 41 \\
\hline & L middle temporal gyrus & 27 & 4.804 & 3.87 & -48 & -67 & 11 \\
\hline & Location not in atlas & 5 & 4.661 & 3.79 & 48 & -49 & 5 \\
\hline & $\mathrm{R}$ middle temporal gyrus & 13 & 4.621 & 3.77 & 60 & -34 & -1 \\
\hline & Location not in atlas & 8 & 4.523 & 3.71 & 39 & -49 & 23 \\
\hline & Location not in atlas & 14 & 4.516 & 3.71 & -27 & -43 & 23 \\
\hline & L PCC & 16 & 4.515 & 3.71 & -6 & -49 & 35 \\
\hline & Location not in atlas & 11 & 4.502 & 3.70 & -33 & -28 & 44 \\
\hline & Location not in atlas & 15 & 4.269 & 3.56 & 15 & -22 & 44 \\
\hline & L MCC & 5 & 4.061 & 3.43 & -6 & -10 & 56 \\
\hline & L postcentral gyrus & 5 & 4.036 & 3.41 & -54 & -22 & 29 \\
\hline & L cerebelum (Crus 1) & 6 & 4.020 & 3.40 & -3 & -85 & -13 \\
\hline & L middle temporal gyrus & 5 & 3.803 & 3.26 & -63 & -46 & 8 \\
\hline \multirow[t]{2}{*}{ Long detour $>$ Shortcut } & L middle occipital gyrus & 1128 & 11.445 & 6 & -21 & -97 & 2 \\
\hline & $\mathrm{R}$ calcarine gyrus & 1135 & 10.233 & 6 & 15 & -94 & 5 \\
\hline
\end{tabular}




\begin{tabular}{|c|c|c|c|c|c|c|c|}
\hline Contrast Name & Region Label & Extent & $t$ Value & $z$ Value & $x$ & $y$ & $z$ \\
\hline & L cerebelum (VII) & 72 & 6.871 & 5 & -6 & -79 & -37 \\
\hline & R IFG (p. orbitalis) & 53 & 6.615 & 5 & 33 & 23 & -4 \\
\hline & L superior medial gyrus & 228 & 6.605 & 5 & -6 & 20 & 47 \\
\hline & R calcarine gyrus & 56 & 6.395 & 5 & 21 & -55 & 14 \\
\hline & R cerebelum (IX) & 184 & 6.166 & 5 & 15 & -46 & -46 \\
\hline & Location not in atlas & 31 & 6.050 & 5 & 27 & -61 & -31 \\
\hline & $\mathrm{R}$ caudate nucleus & 176 & 5.988 & 4 & 15 & 8 & 8 \\
\hline & L superior orbital gyrus & 93 & 5.798 & 4 & -27 & 53 & -1 \\
\hline & $\mathrm{L}$ middle frontal gyrus & 178 & 5.703 & 4 & -27 & 8 & 56 \\
\hline & $\mathrm{R}$ middle frontal gyrus & 70 & 5.271 & 4 & 51 & 20 & 38 \\
\hline & $\mathrm{L}$ middle frontal gyrus & 88 & 5.224 & 4 & -48 & 29 & 35 \\
\hline & R superior frontal gyrus & 66 & 4.941 & 4 & 24 & 14 & 53 \\
\hline & Location not in atlas & 7 & 4.650 & 4 & -3 & 14 & 23 \\
\hline & $\mathrm{R}$ fusiform gyrus & 7 & 4.642 & 4 & 33 & -4 & -34 \\
\hline & R IFG (p. opercularis) & 29 & 4.639 & 4 & 45 & 8 & 20 \\
\hline & R cerebelum (VI) & 31 & 4.588 & 4 & 9 & -76 & -22 \\
\hline & R superior frontal gyrus & 7 & 4.489 & 4 & 27 & 41 & 44 \\
\hline & L IFG (p. orbitalis) & 32 & 4.473 & 4 & -30 & 29 & -1 \\
\hline & L cerebelum (VIII) & 12 & 4.399 & 4 & -36 & -64 & -49 \\
\hline & Location not in atlas & 6 & 4.287 & 4 & 6 & -28 & -4 \\
\hline & L precentral gyrus & 10 & 4.265 & 4 & -39 & 2 & 38 \\
\hline & Location not in atlas & 8 & 4.255 & 4 & -9 & -28 & 2 \\
\hline & R cerebelum (VIII) & 13 & 4.146 & 3 & 21 & -73 & -46 \\
\hline & Location not in atlas & 14 & 4.141 & 3 & 18 & -37 & -43 \\
\hline & $\mathrm{R}$ insula lobe & 6 & 4.061 & 3 & 39 & -19 & 11 \\
\hline & L posterior-medial frontal & 7 & 4.022 & 3 & -3 & 14 & 68 \\
\hline & R superior frontal gyrus & 6 & 3.920 & 3 & 24 & 14 & 68 \\
\hline & R superior frontal gyrus & 5 & 3.850 & 3 & 24 & 32 & 56 \\
\hline & L temporal pole & 8 & 3.765 & 3 & -39 & 14 & -13 \\
\hline \multirow[t]{8}{*}{ Long detour $>$ Short detour } & R cerebelum (VI) & 408 & 7.734 & 5 & 9 & -82 & -16 \\
\hline & L calcarine gyrus & 349 & 6.550 & 5 & -15 & -58 & 11 \\
\hline & L cerebelum (Crus 1) & 104 & 6.080 & 5 & -24 & -85 & -16 \\
\hline & L ACC & 98 & 5.456 & 4 & -3 & 32 & 32 \\
\hline & Location not in atlas & 11 & 5.311 & 4 & -27 & 2 & -10 \\
\hline & R cerebelum (VIII) & 12 & 5.109 & 4 & 21 & -55 & -43 \\
\hline & Location not in atlas & 12 & 5.081 & 4 & 15 & -25 & -31 \\
\hline & Location not in atlas & 10 & 4.850 & 4 & 6 & 5 & -13 \\
\hline
\end{tabular}




\begin{tabular}{|c|c|c|c|c|c|c|c|}
\hline Contrast Name & Region Label & Extent & $t$ Value & $z$ Value & $x$ & $y$ & $z$ \\
\hline & L precuneus & 14 & 4.780 & 4 & -9 & -70 & 44 \\
\hline & L cerebelum (Crus 1) & 21 & 4.776 & 4 & -39 & -49 & -34 \\
\hline & R superior frontal gyrus & 5 & 4.765 & 4 & 27 & 38 & 41 \\
\hline & Location not in atlas & 38 & 4.730 & 4 & 6 & -16 & -10 \\
\hline & L cerebelum (VI) & 11 & 4.653 & 4 & -27 & -61 & -31 \\
\hline & R cerebelum (IX) & 71 & 4.567 & 4 & 6 & -55 & -31 \\
\hline & $\mathrm{R}$ thalamus & 6 & 4.506 & 4 & 21 & -19 & 8 \\
\hline & R fusiform gyrus & 8 & 4.382 & 4 & 36 & -55 & -16 \\
\hline & L linual gyrus & 6 & 4.378 & 4 & -21 & -55 & -7 \\
\hline & $\mathrm{L}$ angular gyrus & 5 & 4.297 & 4 & -45 & -70 & 29 \\
\hline & Location not in atlas & 16 & 4.247 & 4 & -15 & -52 & -22 \\
\hline & $\mathrm{R}$ middle occipital gyrus & 30 & 4.235 & 4 & 36 & -79 & 23 \\
\hline & Location not in atlas & 15 & 4.192 & 4 & -27 & -73 & 17 \\
\hline & $\mathrm{R}$ mid orbital gyrus & 5 & 4.135 & 3 & 3 & 38 & -7 \\
\hline & Cerebellar vermis (3) & 21 & 4.117 & 3 & 6 & -43 & -1 \\
\hline & R IFG (p. triangularis) & 16 & 4.068 & 3 & 45 & 20 & 32 \\
\hline & R superior medial gyrus & 27 & 4.064 & 3 & 3 & 53 & 20 \\
\hline & L precuneus & 7 & 3.946 & 3 & -9 & -61 & 47 \\
\hline \multirow[t]{20}{*}{ False shortcut toward $>$ Shortcut } & $\mathrm{R}$ inferior occipital gyrus & 280 & 8.880 & 5.59 & 30 & -91 & -10 \\
\hline & L linual gyrus & 321 & 8.062 & 5.32 & -24 & -94 & -10 \\
\hline & Location not in atlas & 238 & 7.956 & 5.28 & 39 & 11 & 26 \\
\hline & R superior orbital gyrus & 52 & 5.793 & 4.39 & 33 & 56 & 2 \\
\hline & $\mathrm{R}$ inferior parietal lobule & 83 & 5.743 & 4.36 & 33 & -55 & 47 \\
\hline & R MCC & 24 & 5.297 & 4.14 & 6 & 32 & 35 \\
\hline & Location not in atlas & 113 & 5.220 & 4.10 & 27 & -64 & 41 \\
\hline & R IFG (p. orbitalis) & 11 & 4.874 & 3.91 & 30 & 23 & -4 \\
\hline & $\mathrm{R}$ inferior temporal gyrus & 30 & 4.819 & 3.88 & 45 & -61 & -10 \\
\hline & L middle frontal gyrus & 36 & 4.710 & 3.82 & -33 & 59 & 14 \\
\hline & L superior occipital gyrus & 32 & 4.704 & 3.81 & -18 & -70 & 44 \\
\hline & $\mathrm{L}$ cerebelum $(\mathrm{X})$ & 16 & 4.676 & 3.80 & -18 & -34 & -37 \\
\hline & $\mathrm{R}$ cerebelum (VIII) & 15 & 4.659 & 3.79 & 12 & -73 & -31 \\
\hline & L middle frontal gyrus & 17 & 4.634 & 3.77 & -33 & 8 & 62 \\
\hline & L superior medial gyrus & 38 & 4.349 & 3.61 & 3 & 20 & 56 \\
\hline & L middle frontal gyrus & 22 & 4.279 & 3.56 & -36 & 5 & 38 \\
\hline & L cerebelum (III) & 5 & 4.154 & 3.49 & -6 & -49 & -16 \\
\hline & R IFG (p. triangularis) & 16 & 4.114 & 3.46 & 45 & 35 & 17 \\
\hline & R superior medial gyrus & 5 & 3.997 & 3.39 & 9 & 32 & 62 \\
\hline & L IFG (p. triangularis) & 11 & 3.966 & 3.37 & -48 & 29 & 32 \\
\hline
\end{tabular}


APPENDIX A (continued)

\begin{tabular}{|c|c|c|c|c|c|c|c|}
\hline Contrast Name & Region Label & Extent & $t$ Value & $z$ Value & $x$ & $y$ & $z$ \\
\hline & Location not in atlas & 6 & 3.951 & 3.36 & -9 & -43 & -37 \\
\hline & L cerebelum (VII) & 13 & 3.940 & 3.35 & -6 & -76 & -31 \\
\hline & L superior frontal gyrus & 6 & 3.886 & 3.31 & -15 & 17 & 68 \\
\hline \multirow[t]{5}{*}{ False shortcut toward $<$ Shortcut } & L postcentral gyrus & 95 & 9.192 & 5.69 & -33 & -31 & 47 \\
\hline & L linual gyrus & 480 & 6.417 & 4.68 & -9 & -76 & -1 \\
\hline & R cuneus & 19 & 4.976 & 3.97 & 18 & -82 & 26 \\
\hline & L posterior-medial frontal & 6 & 4.438 & 3.66 & -9 & -4 & 59 \\
\hline & L postcentral gyrus & 14 & 4.358 & 3.61 & -51 & -19 & 26 \\
\hline \multirow[t]{24}{*}{ False shortcut toward correct $>$ Incorrect } & $\mathrm{R}$ middle frontal gyrus & 222 & 10.577 & 5.80 & 27 & -1 & 56 \\
\hline & $\mathrm{R}$ calcarine gyrus & 423 & 9.681 & 5.57 & 15 & -79 & 8 \\
\hline & R fusiform gyrus & 65 & 8.497 & 5.24 & 30 & -43 & -10 \\
\hline & R IFG (p. orbitalis) & 86 & 8.151 & 5.13 & 33 & 26 & -4 \\
\hline & L middle frontal gyrus & 135 & 7.245 & 4.83 & -21 & 2 & 53 \\
\hline & L superior occipital gyrus & 145 & 7.044 & 4.76 & -9 & -97 & 14 \\
\hline & $\mathrm{R}$ postcentral gyrus & 236 & 6.900 & 4.70 & 63 & -22 & 44 \\
\hline & L posterior-medial frontal & 112 & 6.087 & 4.38 & -6 & 14 & 50 \\
\hline & L IFG (p. orbitalis) & 73 & 5.976 & 4.33 & -33 & 23 & 2 \\
\hline & L superior occipital gyrus & 45 & 5.936 & 4.31 & -15 & -73 & 44 \\
\hline & Location not in atlas & 5 & 5.729 & 4.22 & -6 & -25 & -7 \\
\hline & L inferior parietal lobule & 110 & 5.665 & 4.19 & -48 & -40 & 47 \\
\hline & L fusiform gyrus & 23 & 5.619 & 4.17 & -30 & -46 & -7 \\
\hline & R IFG (p. triangularis) & 44 & 5.430 & 4.08 & 42 & 11 & 29 \\
\hline & L cerebelum (VI) & 10 & 5.102 & 3.92 & -27 & -61 & -31 \\
\hline & $\mathrm{R}$ inferior temporal gyrus & 14 & 4.955 & 3.85 & 48 & -46 & -13 \\
\hline & L calcarine gyrus & 14 & 4.698 & 3.71 & -15 & -73 & 11 \\
\hline & R cerebelum (IX) & 7 & 4.402 & 3.55 & 15 & -52 & -49 \\
\hline & L calcarine gyrus & 12 & 4.361 & 3.52 & -15 & -67 & 23 \\
\hline & Location not in atlas & 11 & 4.203 & 3.43 & -3 & -43 & -37 \\
\hline & L precentral gyrus & 9 & 4.071 & 3.35 & -42 & 2 & 35 \\
\hline & Location not in atlas & 6 & 3.999 & 3.31 & -30 & -70 & -55 \\
\hline & L cerebelum (VIII) & 5 & 3.932 & 3.27 & -15 & -73 & -49 \\
\hline & Location not in atlas & 6 & 3.754 & 3.16 & 0 & -25 & -1 \\
\hline \multirow[t]{3}{*}{ False shortcut toward correct $<$ Incorrect } & $\mathrm{L}$ angular gyrus & 100 & 8.233 & 5.16 & -39 & -58 & 26 \\
\hline & Location not in atlas & 90 & 6.291 & 4.46 & 36 & -49 & 26 \\
\hline & L PCC & 121 & 6.132 & 4.40 & -6 & -55 & 35 \\
\hline
\end{tabular}




\begin{tabular}{|c|c|c|c|c|c|c|c|}
\hline Contrast Name & Region Label & Extent & $t$ Value & $z$ Value & $x$ & $y$ & $z$ \\
\hline & L rolandic operculum & 13 & 5.641 & 4.18 & -36 & -37 & 20 \\
\hline & $\mathrm{R}$ caudate nucleus & 22 & 5.410 & 4.07 & 21 & 17 & 20 \\
\hline & Location not in atlas & 32 & 5.122 & 3.93 & -21 & 5 & 26 \\
\hline & Location not in atlas & 7 & 4.958 & 3.85 & -33 & -64 & 8 \\
\hline & $\mathrm{R}$ caudate nucleus & 23 & 4.898 & 3.82 & 21 & -1 & 29 \\
\hline & L middle temporal gyrus & 11 & 4.878 & 3.81 & -60 & -58 & 8 \\
\hline & Location not in atlas & 8 & 4.874 & 3.80 & 21 & -31 & 53 \\
\hline & Location not in atlas & 12 & 4.828 & 3.78 & 3 & -82 & -4 \\
\hline & $\mathrm{R}$ angular gyrus & 13 & 4.560 & 3.64 & 54 & -64 & 32 \\
\hline & R superior temporal gyrus & 25 & 4.529 & 3.62 & 54 & -7 & 8 \\
\hline & $\mathrm{R}$ inferior occipital gyrus & 6 & 4.528 & 3.62 & 39 & -85 & -7 \\
\hline & R MCC & 8 & 4.011 & 3.32 & 15 & -19 & 50 \\
\hline & Location not in atlas & 5 & 3.858 & 3.22 & -15 & -16 & 41 \\
\hline
\end{tabular}

$p<.001$ (uncorrected), minimum of five contiguous voxels. The table shows all local maxima separated by more than 20 mm. Regions were automatically labeled using the AnatomyToolbox atlas using BSPMVIEW (https://github.com/spunt/bspmview). IFG = inferior-frontal gyrus; PCC = posterior-cingulate cortex; MCC = middle-cingulate cortex.

\section{Acknowledgments}

We thank Mate Lengyel for advice on the experimental design and Gareth Barnes and the members of Wellcome Trust Centre for Neuroimaging (University College London) MEG meetings for input into data analysis. This work was supported by the Wellcome Trust (grant 094850/Z/10/Z) and James S. McDonnell Foundation to H. J. S. and the Gatsby Charitable Foundation and the Max Planck Society (P. D.).

Reprint requests should be sent to Eva Zita Patai, Institute of Behavioral Neuroscience, University College London, 26 Bedford Way, London, WC1H 0AP, United Kingdom, or via e-mail: e.patai@ucl.ac.uk, or Hugo J. Spiers, Institute of Behavioral Neuroscience, University College London, 26 Bedford Way, London, WC1H 0AP, United Kingdom, or via e-mail: h.spiers@ucl.ac.uk.

\section{REFERENCES}

Alvernhe, A., Save, E., \& Poucet, B. (2011). Local remapping of place cell firing in the Tolman detour task. European Journal of Neuroscience, 33, 1696-1705.

Alvernhe, A., Van Cauter, T., Save, E., \& Poucet, B. (2008). Different CA1 and CA3 representations of novel routes in a shortcut situation. Journal of Neuroscience, 28, 7324-7333.

Bohbot, V. D., Copara, M. S., Gotman, J., \& Ekstrom, A. D. (2017). Low-frequency theta oscillations in the human hippocampus during real-world and virtual navigation. Nature Communications, 8, 14415.

Bush, D., Bisby, J. A., Bird, C. M., Gollwitzer, S., Rodionov, R., Diehl, B., et al. (2017). Human hippocampal theta power indicates movement onset and distance travelled. Proceedings of the National Academy of Sciences, U.S.A., 114, 12297-12302.

Buzsáki, G. (2005). Theta rhythm of navigation: Link between path integration and landmark navigation, episodic and semantic memory. Hippocampus, 15, 827-840.
Caplan, J. B., Madsen, J. R., Raghavachari, S., \& Kahana, M. J. (2001). Distinct patterns of brain oscillations underlie two basic parameters of human maze learning. Journal of Neurophysiology, 86, 368-380.

Cavanagh, J. F., \& Frank, M. J. (2014). Frontal theta as a mechanism for cognitive control. Trends in Cognitive Sciences, 18, 414-421.

Chakravarthy, V. S., \& Balasubramani, P. P. (2015). Basal ganglia system as an engine for exploration. In D. Jaeger \& R. Jung (Eds.), Encyclopedia of computational neuroscience (pp. 1-15). New York: Springer.

Chapuis, N. (1987). Detour and shortcut abilities in several species of mammals. In P. Ellen \& C. Thinus-Blanc (Eds.), Cognitive processes and spatial orientation in animal and man (Vol. 1, pp. 97-106). Dordrecht, The Netherlands: Springer.

Chapuis, N., Durup, M., \& Thinus-Blanc, C. (1987). The role of exploratory experience in a shortcut task by golden hamsters (Mesocricetus auratus). Animal Learning $\mathcal{E}$ Behavior, 15, 174-178.

Conner, C. R., Ellmore, T. M., Pieters, T. A., DiSano, M. A., \& Tandon, N. (2011). Variability of the relationship between electrophysiology and BOLD-fMRI across cortical regions in humans. Journal of Neuroscience, 31, 12855-12865.

Cornwell, B. R., Johnson, L. L., Holroyd, T., Carver, F. W., \& Grillon, C. (2008). Human hippocampal and parahippocampal theta during goal-directed spatial navigation predicts performance on a virtual Morris water maze. Journal of Neuroscience, 28, 5983-5990.

Dickinson, A., \& Balleine, B. (2002). The role of learning in the operation of motivational systems. In R. Gallistel (Ed.), Stevens' handbook of experimental psychology (Vol. 3, pp. 497-534). New York: Wiley.

Ekstrom, A. D., Spiers, H. J., Bohbot, V. D., \& Rosenbaum, R. S. (2018). Human spatial navigation. Princeton, NJ: Princeton University Press.

Epstein, R. A., Patai, E. Z., Julian, J. B., \& Spiers, H. J. (2017). The cognitive map in humans: Spatial navigation and beyond. Nature Neuroscience, 20, 1504-1513. 
Eschmann, K. C. J., Bader, R., \& Mecklinger, A. (2018). Topographical differences of frontal-midline theta activity reflect functional differences in cognitive control abilities. Brain and Cognition, 123, 57-64.

Hanslmayr, S., Staresina, B. P., \& Bowman, H. (2016). Oscillations and episodic memory: Addressing the synchronization/desynchronization conundrum. Trends in Neurosciences, 39, 16-25.

Hanslmayr, S., Staudigl, T., \& Fellner, M.-C. (2012). Oscillatory power decreases and long-term memory: The information via desynchronization hypothesis. Frontiers in Human Neuroscience, 6, 74 .

Hartley, T., Lever, C., Burgess, N., \& O'Keefe, J. (2014). Space in the brain: How the hippocampal formation supports spatial cognition. Philosophical Transactions of the Royal Society of London, Series B: Biological Sciences, 369, 20120510.

Hassabis, D., \& Maguire, E. A. (2007). Deconstructing episodic memory with construction. Trends in Cognitive Sciences, 11, 299-306.

Hasselmo, M. E., Hay, J., Ilyn, M., \& Gorchetchnikov, A. (2002). Neuromodulation, theta rhythm and rat spatial navigation. Neural Networks, 15, 689-707.

Hasselmo, M. E., Hinman, J. R., Dannenberg, H., \& Stern, C. E. (2017). Models of spatial and temporal dimensions of memory. Current Opinion in Behavioral Sciences, 17, $27-33$.

Hauser, T. U., Iannaccone, R., Stämpfli, P., Drechsler, R., Brandeis, D., Walitza, S., et al. (2014). The feedback-related negativity (FRN) revisited: New insights into the localization, meaning and network organization. Neuroimage, 84 , 159-168.

Hegarty, M., Montello, D. R., Richardson, A. E., Ishikawa, T., \& Lovelace, K. (2006). Spatial abilities at different scales: Individual differences in aptitude-test performance and spatial-layout learning. Intelligence, 34, 151-176.

Holroyd, C. B., \& Coles, M. G. H. (2002). The neural basis of human error processing: Reinforcement learning, dopamine, and the error-related negativity. Psychological Review, 109, 679-709.

Howard, L. R., Javadi, A.-H., Yu, Y., Mill, R. D., Morrison, L. C., Knight, R., et al. (2014). The hippocampus and entorhinal cortex encode the path and Euclidean distances to goals during navigation. Current Biology, 24, 1331-1340.

Iaria, G., Fox, C. J., Chen, J.-K., Petrides, M., \& Barton, J. J. S. (2008). Detection of unexpected events during spatial navigation in humans: Bottom-up attentional system and neural mechanisms. European Journal of Neuroscience, 27 , $1017-1025$.

Jacobs, J. (2014). Hippocampal theta oscillations are slower in humans than in rodents: Implications for models of spatial navigation and memory. Philosophical Transactions of the Royal Society of London, Series B: Biological Sciences, 369, 20130304

Javadi, A.-H., Emo, B., Howard, L. R., Zisch, F. E., Yu, Y., Knight, R., et al. (2017). Hippocampal and prefrontal processing of network topology to simulate the future. Nature Communications, 8, 14652.

Jocham, G., Furlong, P. M., Kröger, I. L., Kahn, M. C., Hunt, L. T., \& Behrens, T. E. J. (2014). Dissociable contributions of ventromedial prefrontal and posterior parietal cortex to value-guided choice. Neuroimage, 100, 498-506.

Kaplan, R., Bush, D., Bisby, J. A., Horner, A. J., Meyer, S. S., \& Burgess, N. (2017). Medial prefrontal-medial temporal theta phase coupling in dynamic spatial imagery. Journal of Cognitive Neuroscience, 29, 507-519.

Kaplan, R., Bush, D., Bonnefond, M., Bandettini, P. A., Barnes, G. R., Doeller, C. F., et al. (2014). Medial prefrontal theta phase coupling during spatial memory retrieval. Hippocampus, 24, 656-665.

Kaplan, R., Doeller, C. F., Barnes, G. R., Litvak, V., Düzel, E., Bandettini, P. A., et al. (2012). Movement-related theta rhythm in humans: Coordinating self-directed hippocampal learning. PLoS Biology, 10, e1001267.

Kaplan, R., King, J., Koster, R., Penny, W. D., Burgess, N., \& Friston, K. J. (2017). The neural representation of prospective choice during spatial planning and decisions. PLoS Biology, 15, e1002588.

Maguire, E. A., Burgess, N., Donnett, J. G., Frackowiak, R. S. J., Frith, C. D., \& O'Keefe, J. (1998). Knowing where and getting there: A human navigation network. Science, 280, 921-924.

Maldjian, J. A., Laurienti, P. J., Kraft, R. A., \& Burdette, J. H. (2003). An automated method for neuroanatomic and cytoarchitectonic atlas-based interrogation of fMRI data sets. Neuroimage, 19, 1233-1239.

Namboodiri, V. M. K., Levy, J. M., Mihalas, S., Sims, D. W., \& Hussain Shuler, M. G. (2016). Rationalizing spatial exploration patterns of wild animals and humans through a temporal discounting framework. Proceedings of the National Academy of Sciences, U.S.A., 113, 8747-8752.

O'Doherty, J., Dayan, P., Schultz, J., Deichmann, R., Friston, K., \& Dolan, R. J. (2004). Dissociable roles of ventral and dorsal striatum in instrumental conditioning. Science, 304 , 452-454.

O'Keefe, J., \& Nadel, L. (1978). The bippocampus as a cognitive map. London: Oxford University Press.

Ólafsdóttir, H. F., Barry, C., Saleem, A. B., Hassabis, D., \& Spiers, H. J. (2015). Hippocampal place cells construct reward related sequences through unexplored space. eLife, 4, e06063.

Oostenveld, R., Fries, P., Maris, E., \& Schoffelen, J.-M. (2011). FieldTrip: Open source software for advanced analysis of MEG, EEG, and invasive electrophysiological data. Computational Intelligence and Neuroscience, 2011, 156869.

Patai, E. Z., Javadi, A.-H., Ozubko, J. D., O’Callaghan, A., Ji, S., Robin, J., et al. (2019). Hippocampal and retrosplenial goal distance coding after long-term consolidation of a real-world environment. Cerebral Cortex, 29, 2748-2758.

Pfeiffer, B. E., \& Foster, D. J. (2013). Hippocampal place-cell sequences depict future paths to remembered goals. Nature, 497, 74-79.

Poucet, B., Thinus-Blanc, C., \& Chapuis, N. (1983). Route planning in cats, in relation to the visibility of the goal. Animal Behaviour, 31, 594-599.

Rauchs, G., Orban, P., Balteau, E., Schmidt, C., Degueldre, C., Luxen, A., et al. (2008). Partially segregated neural networks for spatial and contextual memory in virtual navigation. Hippocampus, 18, 503-518.

Ribas-Fernandes, J. J. F., Shahnazian, D., Holroyd, C. B., \& Botvinick, M. M. (2019). Subgoal- and goal-related reward prediction errors in medial prefrontal cortex. Journal of Cognitive Neuroscience, 31, 8-23.

Ribas-Fernandes, J. J. F., Solway, A., Diuk, C., McGuire, J. T., Barto, A. G., Niv, Y., et al. (2011). A neural signature of hierarchical reinforcement learning. Neuron, 71, 370-379.

Rosenbaum, R. S., Ziegler, M., Winocur, G., Grady, C. L., \& Moscovitch, M. (2004). "I have often walked down this street before": fMRI studies on the hippocampus and other structures during mental navigation of an old environment. Hippocampus, 14, 826-835.

Scheeringa, R., Fries, P., Petersson, K.-M., Oostenveld, R., Grothe, I., Norris, D. G., et al. (2011). Neuronal dynamics underlying high- and low-frequency EEG oscillations contribute independently to the human BOLD signal. Neuron, 69, 572-583. 
Scheeringa, R., Petersson, K. M., Oostenveld, R., Norris, D. G., Hagoort, P., \& Bastiaansen, M. C. M. (2009). Trial-by-trial coupling between EEG and BOLD identifies networks related to alpha and theta EEG power increases during working memory maintenance. Neuroimage, 44, 1224-1238.

Shallice, T. (1982). Specific impairments of planning. Philosophical Transactions of the Royal Society of London, Series B: Biological Sciences, 298, 199-209.

Simon, D. A., \& Daw, N. D. (2011). Neural correlates of forward planning in a spatial decision task in humans. Journal of Neuroscience, 31, 5526-5539.

Snider, J., Plank, M., Lynch, G., Halgren, E., \& Poizner, H. (2013). Human cortical $\theta$ during free exploration encodes space and predicts subsequent memory. Journal of Neuroscience, 33, 15056-15068.RL

Spiers, H. J. (2008). Keeping the goal in mind: Prefrontal contributions to spatial navigation. Neuropsychologia, 46, 2106-2108.

Spiers, H. J., \& Barry, C. (2015). Neural systems supporting navigation. Current Opinion in Behavioral Sciences, 1, 47-55.

Spiers, H. J., \& Gilbert, S. J. (2015). Solving the detour problem in navigation: A model of prefrontal and hippocampal interactions. Frontiers in Human Neuroscience, 9, 125.

Spiers, H. J., \& Maguire, E. A. (2006). Thoughts, behaviour, and brain dynamics during navigation in the real world. Neuroimage, 31, 1826-1840.

Staudigl, T., Vollmar, C., Noachtar, S., \& Hanslmayr, S. (2015). Temporal-pattern similarity analysis reveals the beneficial and detrimental effects of context reinstatement on human memory. Journal of Neuroscience, 35, 5373-5384.

Tolman, E. C. (1948). Cognitive maps in rats and men. Psychological Review, 55, 189-208.
Tolman, E. C., \& Honzik, C. H. (1930). Introduction and removal of reward and maze performance in rats. University of California Publications in Psychology, 4, 257-275.

Tzourio-Mazoyer, N., Landeau, B., Papathanassiou, D., Crivello, F., Etard, O., Delcroix, N., et al. (2002). Automated anatomical labeling of activations in SPM using a macroscopic anatomical parcellation of the MNI MRI single-subject brain. Neuroimage, 15, 273-289.

Vass, L. K., Copara, M. S., Seyal, M., Shahlaie, K., Farias, S. T., Shen, P. Y., et al. (2016). Oscillations go the distance: Lowfrequency human hippocampal oscillations code spatial distance in the absence of sensory cues during teleportation. Neuron, 89, 1180-1186.

Viard, A., Doeller, C. F., Hartley, T., Bird, C. M., \& Burgess, N. (2011). Anterior hippocampus and goal-directed spatial decision making. Journal of Neuroscience, 31, 4613-4621.

Watrous, A. J., Fried, I., \& Ekstrom, A. D. (2011). Behavioral correlates of human hippocampal delta and theta oscillations during navigation. Journal of Neurophysiology, 105, 1747-1755.

Weidemann, C. T., Mollison, M. V., \& Kahana, M. J. (2009). Electrophysiological correlates of high-level perception during spatial navigation. Psychonomic Bulletin \& Review, 16, 313-319.

Winocur, G., Moscovitch, M., Rosenbaum, R. S., \& Sekeres, M. (2010). An investigation of the effects of hippocampal lesions in rats on pre- and postoperatively acquired spatial memory in a complex environment. Hippocampus, 20, 1350-1365.

Xu, J., Evensmoen, H. R., Lehn, H., Pintzka, C. W. S., \& Håberg, A. K. (2010). Persistent posterior and transient anterior medial temporal lobe activity during navigation. Neuroimage, 52, 1654-1666.

Yoshida, W., \& Ishii, S. (2006). Resolution of uncertainty in prefrontal cortex. Neuron, 50, 781-789. 